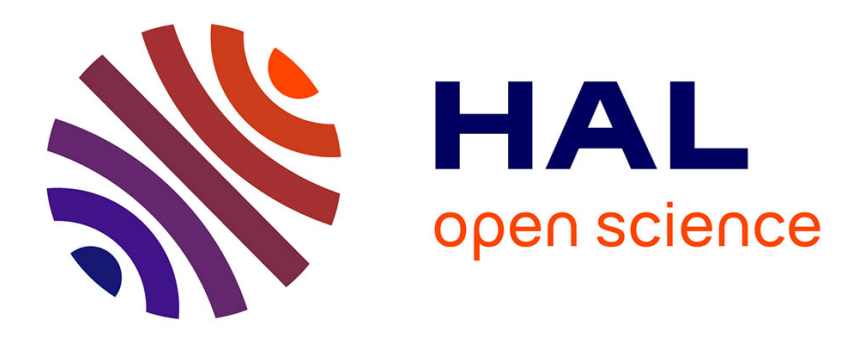

\title{
Spectrum of ultrametric Banach algebras of strictly differentiable functions
}

\author{
Alain Escassut, Nicolas Maïnetti
}

\section{To cite this version:}

Alain Escassut, Nicolas Maïnetti. Spectrum of ultrametric Banach algebras of strictly differentiable functions. Contemporary mathematics, 2018, Advances in Ultrametric Analysis, 704, pp.139-159. 10.1090/conm/704/14165 . hal-01918238

\section{HAL Id: hal-01918238 \\ https://hal.uca.fr/hal-01918238}

Submitted on 10 Nov 2018

HAL is a multi-disciplinary open access archive for the deposit and dissemination of scientific research documents, whether they are published or not. The documents may come from teaching and research institutions in France or abroad, or from public or private research centers.
L'archive ouverte pluridisciplinaire HAL, est destinée au dépôt et à la diffusion de documents scientifiques de niveau recherche, publiés ou non, émanant des établissements d'enseignement et de recherche français ou étrangers, des laboratoires publics ou privés. 


\title{
Spectrum of ultrametric Banach algebras of strictly differentiable functions
}

\author{
Alain Escassut and Nicolas Maïnetti
}

\begin{abstract}
Let $\mathbb{I K}$ be an ultrametric complete field and let $E$ be an open subset of $\mathbb{I K}$ of strictly positive codiameter. Let $\mathcal{D}(E)$ be the Banach $\mathbb{K}$-algebra of bounded strictly differentiable functions from $E$ to $\mathbb{K}$, a notion whose definition is detailed. It is shown that all elements of $\mathcal{D}(E)$ have a derivative that is continuous in $E$. Given a positive number $r>0$, all functions that are bounded and are analytic in all open disks of diameter $r$ are strictly differentiable. Maximal ideals and continuous multiplicative semi-norms on $\mathcal{D}(E)$ are studied by recalling the relation of contiguity on ultrafilters: an equivalence relation. So, the maximal spectrum of $\mathcal{D}(E)$ is in bijection with the set of equivalence classes with respect to contiguity. Every prime ideal of $\mathcal{D}(E)$ is included in a unique maximal ideal and every prime closed ideal of $\mathcal{D}(E)$ is a maximal ideal, hence every continuous multiplicative semi-norm on $\mathcal{D}(E)$ has a kernel that is a maximal ideal. If $\mathbb{K}$ is locally compact, every maximal ideal of $\mathcal{D}(E)$ is of codimension 1. Every maximal ideal of $\mathcal{D}(E)$ is the kernel of a unique continuous multiplicative semi-norm and every continuous multiplicative semi-norm is defined as the limit along an ultrafilter on $E$. Consequently, the set of continuous multiplicative semi-norms defined by points of $E$ is dense in the whole set of all continuous multiplicative semi-norms. The Shilov boundary of $\mathcal{D}(E)$ is equal to the whole set of continuous multiplicative semi-norms. Many results are similar to those concerning algebras of uniformly continuous functions but some specific proofs are required.
\end{abstract}

\section{Introduction and preliminaries:}

Let $\mathbb{K}$ be a field which is complete with respect to an ultrametric absolute value that will be denoted by $\mid$. $\mid$.

Consider a Banach $\mathbb{K}$-algebra $T$. It is well known that the set of maximal ideals is not sufficient to describe spectral properties of $T$ : we have to consider the set of continuous multiplicative semi-norms [4], [5], [7], [8], [9], [10], [11], [12], [13], [14]. Many studies were made on continuous multiplicative semi-norms on algebras of analytic functions, analytic elements and their applications to holomorphic functional calculus [1], [3], [4]. Continuous multiplicative semi-norms of the Banach algebras of bounded continuous functions and those of bounded uniformly continuous functions were studied in [10], [11]. After these studies, it would be natural to look for Banach algebras of derivable functions. Unfortunately, such Banach algebras do not seem to exist, as shows the remark below. This is why it seems interesting to consider strictly differentiable functions.

Definitions and notation: Throughout the paper, we denote by $E$ an open subset of $\mathbb{K}$. Given $a \in \mathbb{K}$ and $r>0$, we set $d(a, r)=\{x \in \mathbb{K}|| x-a \mid \leq r\}$,

\footnotetext{
${ }^{0}$ Keywords: strictly differentiable functions, ultrametric fields.

2000 Mathematics Subject Classification. Primary 46S10 Secondary 12J25 .
} 
$d\left(a, r^{-}\right)=\{x \in \mathbb{K}|| x-a \mid<r\} d_{E}(a, r)=\{x \in E|| x-a \mid \leq r\}$ and $d_{E}\left(a, r^{-}\right)=$ $\{x \in E|| x-a \mid<r\}$.

We denote by $\delta$ the distance between two subsets of $\mathbb{K}$ : given two subsets $B_{1}, B_{2}$ of $\mathbb{K}$, we set $\delta\left(B_{1}, B_{2}\right)=\inf \left\{|x-y| \mid x \in B_{1}, y \in B_{2}\right\}$. We denote by diam the diameter of a subset $B$ of $\mathbb{K}$ and we set $\operatorname{codiam}(B)=\delta(B, \mathbb{K} \backslash B)$. Similarly, given a subset $B$ of $E$, we set $\operatorname{codiam}_{E}(B)=\delta(B, E \backslash B)$. A subset $B$ of $E$ will be said to be uniformly open in $E$ or uniformly open subset of $E$ if $\operatorname{codiam}_{E}(B)>0$.

Given a bounded function $f$ from $E$ to $\mathbb{K}$, we put $\|f\|_{0}=\sup _{x \in E}|f(x)|$.

Given a disk $d\left(a, R^{-}\right)$, we denote by $\mathcal{A}\left(d\left(a, R^{-}\right)\right)$the $\mathbb{K}$-algebra of analytic functions in $d\left(a, R^{-}\right)$i.e. the set of power series $\sum_{n=0}^{\infty} a_{n}(x-a)^{n}$ of radius of convergence $r \geq R$. For every $r \in] 0, s]$, we call $r$-analytic function a function from $E$ to $\mathbb{K}$ whose restriction in all disks $d\left(a, r^{-}\right) \subset E$ belongs to $\mathcal{A}\left(d\left(a, r^{-}\right)\right)$and we denote by $\mathcal{A}_{b}(E, r)$ the set of $r$-analytic functions bounded in $E$.

Let $D=\{(x, x) \mid x \in E\}$ and let $\mathcal{D}(E)$ be the $\mathbb{K}$-vector space of bounded functions $f$ from $E$ to $\mathbb{K}$ such that the mapping $\phi$ defined in $(E \times E) \backslash D$ into $\mathbb{K}$ as $\phi(x, y)=\frac{f(x)-f(y)}{x-y}$ is bounded by a bound $M_{f}$ and expands to a continuous function from $E \times E$ to $\mathbb{K}$. The functions $f \in \mathcal{D}(E)$ will be called the strictly differentiable functions from $E$ to $\mathbb{I K}$. Given $f \in \mathcal{D}(E)$, we put $\|f\|_{1}=\sup _{(x, y) \in(E \times E) \backslash D} \phi(x, y)$ and we check that $\|.\|_{1}$ is another $\mathbb{K}$-vector space norm on $\mathcal{D}(E)$. Finally we put $\|f\|=\max \left(\|f\|_{0},\|f\|_{1}\right)$.

Remark 1: A uniformly open subset in $\mathbb{K}$ is open and closed.

Remark 2: Suppose $\mathbb{K}$ is algebraically closed and let $E=d(0,1)$. For every $r \in[0,1] \cap|\mathbb{K}|$, we denote by $\xi(r)$ an element $b$ of $E$ such that $|b|^{2}=r$. The set $E \backslash\{0\}$ obviously admits a partition of the form $\left\{d\left(a_{j},\left|a_{j}\right|^{-}\right)_{j \in I}\right\}$.

Now, let $f$ be the function defined in $E$ in the following way. Given $x \in$ $d\left(a_{j},\left|a_{j}\right|^{-}\right)$, we put $f(x)=\xi\left(\left|a_{j}\right|\right)$ and $f(0)=0$. In this way, $f$ is constant in each disk $d\left(a_{j},\left|a_{j}\right|^{-}\right), a \in E$ and therefore $f$ has a derivative equal to 0 at each point $a \in E \backslash\{0\}$ but $f$ has no derivative at 0 because

$$
\lim _{x \rightarrow 0}\left|\frac{f(x)}{x}\right|=\lim _{x \rightarrow 0} \frac{\sqrt{|x|}}{|x|}=+\infty .
$$

Now, let us take a decreasing sequence $\left(r_{n}\right)_{n \in \mathbb{N}}$ in $|\mathbb{K}|$, of limit 0 and for each $n \in \mathbb{N}$, let $f_{n}$ be the function defined in $E$ by $f_{n}(x)=f(x)$ for every $x \in E \backslash d\left(0, r_{n}\right)$ and $f_{n}(x)=\xi\left(a_{j}\right)$ for every $x \in d\left(0, r_{n}\right)$, with $a_{j} \in d\left(0, r_{n}\right)$. Thus, we can check that $f_{n}$ has a derivative equal to 0 in all $E$. Therefore, the sequence $\left(f_{n}^{\prime}\right)_{n \in \mathbb{N}}$ trivially is uniformly convergent to the function that is identically zero in all $E$.

On the other hand, consider $\left\|f_{n}-f\right\|_{0}$. By construction, we check $|f(x)| \leq \sqrt{r_{n}}$ for every $x \in d\left(0, r_{n}\right)$ and hence $\left|f(x)-f_{n}(x)\right| \leq \sqrt{r_{n}}$ for every $x \in d\left(0, r_{n}\right)$. But since $f_{n}(x)=f(x)$ for every $x \in E \backslash d\left(0, r_{n}\right)$, we derive $\left\|f_{n}-f\right\|_{0} \leq \sqrt{r_{n}}$. Consequently, the sequence $\left(f_{n}\right)_{n \in \mathbb{N}}$ is uniformly convergent in $E$ to $f$. And $f$ is not derivable at 0 , although the sequence $\left(f_{n}^{\prime}\right)_{n \in \mathbb{N}}$ is also uniformly convergent in E. 
Theorems 1 and 2 are designed to recall properties that we can also find in [16], with some differences on hypotheses or definitions.

Theorem 1: Every function $f \in \mathcal{D}(E)$ is uniformly continuous, derivable in $E$ and $f^{\prime}$ is bounded and continuous in $E$. Moreover, if $E$ is compact, then a function from $E$ to $\mathbb{K}$ belongs to $\mathcal{D}(E)$ if and only if for every $a \in E, \frac{f(x)-f(y)}{x-y}$ has a limit when $x$ and $y$ tend to a separately, while being distinct.

Proof. Let $f \in \mathcal{D}(E)$. Then $f$ is Lipschitzian and hence uniformly continuous in $E$. Next, for every $a \in E, \frac{f(x)-f(y)}{x-y}$ has a limit $l(a)$ when $x$ and $y$ tend to $a$ separately, while being distinct. For each $\epsilon>0$ and let $r(\epsilon)>0$ be such that $\left|\frac{f(x)-f(y)}{x-y}-l\right| \leq \epsilon$ for all $x, y \in d_{E}(a, r(\epsilon)), x \neq a, y \neq a, x \neq y$. Without loss of generality, we can assume $|x-a|=r(\epsilon) \geq|y-a|$. Now, if $y$ tends to $a$, the inequality remains and since $f$ is continuous, we obtain $\left|\frac{f(x)-f(a)}{x-a}-l(a)\right| \leq \epsilon$. Consequently, $\lim _{x \rightarrow a} \frac{f(x)-f(a)}{x-a}=l(a)$. And hence $\frac{f(x)-f(y)}{x-y}$ has a limit when $x$ and $y$ tend to $a$ separately, while being distinct, even if $x$ or $y$ is equal to $a$. Particularly, $f$ has a derivative equal to $l(a)$ at each point $a \in E$.

By definition $\left|\frac{f(x)-f(y)}{x-y}\right|$ is bounded in $E$, hence, so is $\left|\frac{f(x)-f(a)}{x-a}\right|$ and hence so is $\left|f^{\prime}(x)\right|$. Finally, let us take $b \in d_{E}(a, r(\epsilon))$. We have $\mid \frac{f(b)-f(a)}{b-a}-$ $f^{\prime}(a) \mid \leq \epsilon$ and $\left|\frac{f(y)-f(x)}{y-x}-f^{\prime}(a)\right| \leq \epsilon$ for all $x, y \in d_{E}(a, r(\epsilon))$, hence $\mid \frac{f(y)-f(b)}{y-b}-$ $f^{\prime}(a) \mid \leq \epsilon$ for every $y \in d_{E}(a, r(\epsilon))$ hence $\left|f^{\prime}(b)-f^{\prime}(a)\right| \leq \epsilon$, which proves that $f^{\prime}$ is continuous.

Now, suppose that $E$ is compact and let $f$ be a function from $E$ to $\mathbb{K}$ such that for every $a \in E, \frac{f(x)-f(y)}{x-y}$ has a limit $l(a)$ when $x$ and $y$ tend to $a$ separately, while being distinct.

We will check that $\left|\frac{f(x)-f(y)}{x-y}\right|$ is bounded by a number $M_{f}$. Suppose that it is not bounded. Then, there exists a sequence of pairs $\left(x_{n}, y_{n}\right)_{n \in \mathbb{N}}$ in $E \times E$ such that $\lim _{n \rightarrow+\infty}\left|\frac{f\left(x_{n}\right)-f\left(y_{n}\right)}{x_{n}-y_{n}}\right|=+\infty$. Since $E$ is compact we can extract from the sequence $\left(x_{n}, y_{n}\right)_{n \in \mathbb{N}}$ a subsequence converging to a pair $(a, b) \in E \times E$. So, without loss of generality, we can assume that the sequence $\left(x_{n}, y_{n}\right)_{n \in \mathbb{N}}$ converges to $(a, b)$. If $a \neq b$, then we have a contradiction due to the continuity of $f$. And if $a=b$, then we have a contradiction due to the very hypothesis. In the same way, by fixing $y$, this proves that $f$ is bounded in $E$.

Remark 3: A function $f$ from $E$ to $\mathbb{K}$ which is derivable with a continuous derivative is not automatically strictly differentiable. Indeed, suppose $E$ has an infinite residue class field. We can find a sequence $\left(a_{n}\right)_{n \in \mathbb{N}}$ such that $\left|a_{n}\right|=\mid a_{n}-$ $a_{m} \mid=1$ for all $n \neq m$. Now take a sequence $\left(r_{n}\right)_{n \in \mathbb{N}}$ of $\mathbb{R}^{+}$with $\lim _{n \rightarrow+\infty} r_{n}=0$. 
Then the set $E \backslash \bigcup_{n=0}^{\infty} d\left(a_{n}, r_{n}^{-}\right)$is open. Now, take $b_{n} \in d\left(a_{n}, r_{n}\right)$ such that $\mid a_{n}-$ $b_{n} \mid=r_{n}$ for every $n \in \mathbb{N}$.

We can define a function $f$ from $E$ to $\mathbb{K}$ such that $f(x)=0$ for all $x \in$ $\bigcup_{n=0}^{\infty} d\left(a_{n}, r_{n}^{-}\right)$and $f(x)=1$ for every $x \in E \backslash \bigcup_{n=0}^{\infty} d\left(a_{n}, r_{n}^{-}\right)$. Of course, $f$ is derivable and $f^{\prime}$ is continuous in $E$. However, $\left|\frac{f\left(a_{n}\right)-f\left(b_{n}\right)}{a_{n}-b_{n}}\right|=\frac{1}{r_{n}}$ and therefore $\left|\frac{f(x)-f(y)}{x-y}\right|$ is not bounded in $E$.

Theorem 2: $\quad \|$. $\|$ is a norm of $\mathbb{K}$-vector space on $\mathcal{D}(E)$ and $\mathcal{D}(E)$ is complete for that norm.

Proof. Both $\|$. $\|_{0}$ and $\|.\|_{1}$ are norms of $\mathbb{K}$-vector space, hence so is $\|$. $\|$. Let us show that $\mathcal{D}(E)$ is complete for that norm.

Let $g$ be a function from $E$ to $\mathbb{K}$ that belongs to the completion of $\mathcal{D}(E)$ with respect to the norm $\|$. $\|$. For all $\epsilon>0$, there exists $f_{\epsilon} \in \mathcal{D}(E)$ such that $\left\|f_{\epsilon}-g\right\| \leq \epsilon$. Since $f_{\epsilon}$ is bounded, so is $g$. Next, we check that $\left\|f_{\epsilon}-g\right\|_{1} \leq \epsilon$, hence

$$
\left|\frac{\left(f_{\epsilon}-g\right)(x)-\left(f_{\epsilon}-g\right)(y)}{x-y}\right| \leq \epsilon
$$

for all $x, y \in(E \times E) \backslash D$. Therefore since $\left|\frac{f_{\epsilon}(x)-f_{\epsilon}(y)}{x-y}\right|$ is bounded by $\left\|f_{\epsilon}\right\|_{1}$ in $(E \times E) \backslash D$, so is $\left|\frac{g(x)-g(y)}{x-y}\right|$. Now we have to check that $\left|\frac{g(x)-g(y)}{x-y}\right|$ expands to a continuous function from $E \times E$ to $\mathbb{I K}$. Let $(a, a) \in D$ and let us fix $\epsilon>0$. By definition, $\left|\frac{f_{\epsilon}(x)-f_{\epsilon}(y)}{x-y}\right|$ has a limit $\left(f_{\epsilon}\right)^{\prime}(a)$ when $x$ and $y$ tend to $a$. Consequently, there exists a disk $d_{E}(a, r)$ in $E$ such that for all $x, y \in d_{E}(a, r), x \neq y$,

$$
\left|\frac{f_{\epsilon}(x)-f_{\epsilon}(y)}{x-y}-\left(f_{\epsilon}\right)^{\prime}(a)\right| \leq \epsilon
$$

But since $\left\|f_{\epsilon}-g\right\|_{1} \leq \epsilon$, we have

$$
\left|\frac{\left(f_{\epsilon}(x)-g(x)\right)-\left(f_{\epsilon}(y)-g(y)\right)}{x-y}\right| \leq \epsilon
$$

for all $x, y \in d_{E}(a, r)$. Consequently, by (1) we derive (2) for all $x, y \in d_{E}(a, r), x \neq$ $y$ :

$$
\left|\frac{g(x)-g(y)}{x-y}-\left(f_{\epsilon}\right)^{\prime}(a)\right| \leq \epsilon .
$$

Now, let us take $\omega \in] 0, \epsilon\left[\right.$ and take $f_{\omega} \in \mathcal{D}(E)$ such that $\left\|f_{\omega}-g\right\| \leq \omega$. There exists a disk $d_{E}(a, s)$ in $E$, with $0<s \leq r$ such that

$$
\left|\frac{f_{\omega}(x)-f_{\omega}(y)}{x-y}-\left(f_{\omega}\right)^{\prime}(a)\right| \leq \omega
$$

for all $x, y \in d_{E}(a, s), x \neq y$. Consequently, when $x, y \in d_{E}(a, s)$, we can see that $\left|\left(f_{\omega}\right)^{\prime}(a)-\left(f_{\epsilon}\right)^{\prime}(a)\right| \leq \epsilon$. Therefore each interval $\left[\left|\left(f_{\epsilon}\right)^{\prime}(a)\right|-\epsilon,\left|\left(f_{\epsilon}\right)^{\prime}(a)\right|+\epsilon\right]$ contains $\frac{g(x)-g(y)}{x-y}$ whenever $x, y \in d_{E}(a, s), x \neq y$. Therefore the family of intervals $\left[\left|\left(f_{\epsilon}\right)^{\prime}(a)\right|-\epsilon,\left|\left(f_{\epsilon}\right)^{\prime}(a)\right|+\epsilon\right]$ is a basis of a Cauchy filter whose limit $L(a)$, 
by (2), is the limit of $\frac{g(x)-g(y)}{x-y}$ when $x$ and $y$ tend to $a$, which finishes the proof that $g$ belongs to $\mathcal{D}(E)$.

Theorem 3: $\mathcal{D}(E)$ is a $\mathbb{K}$-algebra and $\|$.$\| is a norm of \mathbb{K}$-algebra.

Proof. Let $f, g \in \mathcal{D}(E)$. Given $x, y \in E$, we have

$$
\frac{f(x) g(x)-f(y) g(y)}{x-y}=f(x) \frac{g(x)-g(y)}{x-y}+g(y) \frac{f(x)-f(y)}{x-y}
$$

hence the limit at $a$ of $\frac{f(x) g(x)-f(y) g(y)}{x-y}$ when $x, y$ tend to $a$ with $x \neq y$ is $f(a) g^{\prime}(a)+g(a) f^{\prime}(a)$. Next, we can easily check that

$\|f g\|=\max \left(\|f g\|_{0},\|f g\|_{1}\right) \leq \max \left(\|f\|_{0}\|g\|_{0},\|f g\|_{1}\right)$. Now $\|f g\|_{1}=$

$=\sup _{x, y}\left|\frac{f(x) g(x)-f(y) g(y)}{x-y}\right|=\max \left(\sup _{x, y}\left|\frac{f(x)(g(x)-g(y)}{x-y}\right|, \sup _{x, y}\left|\frac{g(y)(f(x)-f(y)}{x-y}\right|\right)$.

Consequently, $\|f g\|_{1} \leq\|f\|\|g\|$ and therefore $\|f g\| \leq\|f\|\|g\|$.

Corollary 3.1: $\mathcal{D}(E)$ is a Banach $\mathbb{K}$-algebra.

Theorem 4: Let $r \in] 0, s]$. Suppose $E$ uniformly open in $\mathbb{K}$, of codiameter $s$ and let $r \in] 0, s]$. Then $\mathcal{A}_{b}(E, r)$ provided with the norm $\|\cdot\|_{E}$ is a Banach $\mathbb{K}$-algebra included in $\mathcal{D}(E)$.

Proof. By definition, $E$ admits a partition by a family of disks $\left(E_{i}\right)_{i \in I}$ of diameter $r$. Now, it is easily seen that $\mathcal{A}(E, r)$ is a $\mathbb{K}$-algebra algebraically and topologically isomorphic to the direct product $\prod_{i \in I} \mathcal{A}\left(E_{i}, r\right)$. Let us show that $\mathcal{A}_{b}(E, r)$ is included in $\mathcal{D}(E)$. Let $f \in \mathcal{A}_{b}(E, r)$, let $M=\|f\|_{E}$ and take $x, y \in E$. We will prove that $\left|\frac{f(x)-f(y)}{x-y}\right|$ is bounded in $E$.

Suppose first that $|x-y| \geq r$. Then $\left|\frac{f(x)-f(y) \mid}{x-y}\right| \leq \frac{M}{r}$. Suppose now that $|x-y|<r$. Then both $x$ and $y$ belong to a same disk $E_{i}$. Clearly, we can assume that $E_{i}$ is the disk $d\left(0, r^{-}\right)$without loss of generality and then, inside that disk, $f(t)$ is a power series $\sum_{n=0}^{+\infty} a_{n} t^{n}$, with $\sup _{n \in \mathbb{N}}\left|a_{n}\right| r^{n} \leq M$. Consequently,

and therefore,

$$
\frac{f(x)-f(y)}{x-y}=\sum_{n=1}^{+\infty} a_{n} \sum_{j=0}^{n-1} x^{j} y^{n-1-j}
$$

$$
\left|\frac{f(x)-f(y)}{x-y}\right| \leq \sup _{n \geq 1}\left|a_{n}\right| r^{n-1} \leq \lim _{s \rightarrow r^{-}} \frac{|f|(s)}{s} \leq \frac{M}{r} .
$$

Consequently, we have proven that

$$
\left|\frac{f(x)-f(y)}{x-y}\right| \leq \frac{M}{r}
$$


for all $x, y \in E$.

Now when $x$ and $y$ approach independently to a same point $a \in E$, both $x$ and $y$ belong to a same disk $E_{i}$ and we can again assume that $E_{i}=d\left(0, r^{-}\right)$. Moreover, without loss of generality, we can also assume that $a=0$. Then we have again

$$
\frac{f(x)-f(y)}{x-y}=\sum_{n=1}^{+\infty} a_{n} \sum_{j=0}^{n-1} x^{j} y^{n-1-j} .
$$

When $x$ and $y$ go separately to 0 , clearly the sum goes to $a_{1}$ which is $f^{\prime}(0)$. Therefore $\mathcal{A}_{b}(E, r)$ is a sub-algebra of $\mathcal{D}(E)$.

On the other hand, by (1), we can see that $\|f\|_{1} \leq \frac{\|f\|_{E}}{r}$ therefore the norms $\|\cdot\|_{E}$ and $\|\cdot\|$ are equivalent on $\mathcal{A}_{b}(E, r)$.

Let sus show that $\mathcal{A}_{b}(E, r)$ is closed with respect to the norm $\|\cdot\|_{E}$ and hence with respect to the norm $\|$. $\|$. Let $\left(f_{n}\right)_{n \in \mathbb{N}}$ be a Cauchy sequence of $\mathcal{A}_{b}(E, r)$. For every disk $d\left(a, r^{-}\right)$, it is a Cauchy sequence of $\mathcal{A}_{b}\left(d\left(a, r^{-}\right)\right)$with respect to the norm $\|\cdot\|_{d\left(a, r^{-}\right)}$, hence the sequence converges to a function $g_{a} \in \mathcal{A}_{b}\left(d\left(a, r^{-}\right)\right)$. Then the function $f$ defined in $E$ as $f(x)=g_{a}(x)$ for every $x \in d\left(a, r^{-}\right)$is clearly the limit of the sequence $\left(f_{n}\right)_{n \in \mathbb{N}}$ with respect to the norm $\|.\|_{E}$. On the other hand, since the sequence $\left(f_{n}\right)$ is a Cauchy sequence of bounded functions, its limit is obviously bounded. Thus, $\mathcal{A}_{b}(E, r)$ is closed in $\mathcal{D}(E)$.

The role of ultrafilters here is essential as in a few previous works [10]. They were deeply studied in [15].

Notation and definitions. Let $\mathcal{F}$ be a filter on $E$. Given a function $f$ from $E$ to $\mathbb{K}$ admitting a limit along $\mathcal{F}$, we will denote by $\lim _{\mathcal{F}} f(x)$ that limit.

Let $U l(E)$ be the set of ultrafilters on $E$. Two filters $\mathcal{F}, \mathcal{G}$ on $E$ will be said to be contiguous if for every $H \in \mathcal{F}, L \in \mathcal{G}$, we have $\delta(H, L)=0$. We shall denote by $(\mathcal{T})$ the relation defined on $U l(E)$ as $\mathcal{U}(T) \mathcal{V}$ if $\mathcal{U}$ and $\mathcal{V}$ are contiguous.

An ultrafilter $\mathcal{U}$ on the set $E$ is said to be principal if it converges to a point $a \in E$.

Remark 4: Let $\mathcal{U}, V$ be contiguous ultrafilters on $E$ and assume $\mathcal{U}$ is convergent. Then $\mathcal{V}$ is convergent and has the same limit as $\mathcal{U}$.

The following Lemma $\mathrm{L}$ is immediate:

Lemma L: Let $X_{1}, \ldots, X_{q}$ be uniformly open subsets of $E$. Then if $\bigcap_{j=1}^{q} X_{j}$ is not empty, it is uniformly open in E.

Theorem 5: Let $X \subset E$ be uniformly open in $E$ and let $u$ be defined as $u(x)=1$ for every $x \in X$ and $u(x)=0$ for every $x \notin X$. Then $u$ belongs to $\mathcal{D}(E)$.

Proof. Let $r=\operatorname{codiam}(X)$. Of course, we have $\frac{u(x)-u(y)}{x-y}=0$ for all $(x, y) \in X \times X, x \neq y$. Finally, let $x \in X, y \in(E \backslash X)$. Then $\left|\frac{u(x)-u(y)}{x-y}\right| \leq \frac{1}{r}$. 
Moreover, if $x$ and $y$ tend to a point $a \in E$, then we can assume that either both belong to $X$ or both belong to $E \backslash X$, hence $\frac{u(x)-u(y)}{x-y}=0$.

Notation: Let $f_{1}, \ldots, f_{q} \in \mathcal{D}(E)$ and let $\epsilon>0$. We set $W\left(f_{1}, \ldots, f_{q}, \epsilon\right)=\{x \in$ $E|| f(x) \mid \leq \epsilon\}$.

Theorem 6: Let $f_{1}, \ldots, f_{q} \in \mathcal{D}(E)$, let $\epsilon>0$. Then if $W\left(f_{1}, \ldots, f_{q}, \epsilon\right)$ is not empty, it is uniformly open in $E$.

Proof. By Lemma L, we can assume $q=1$. So, consider a set $W(f, \epsilon)$. Since $f$ is uniformly continuous, there exists $r(\epsilon)>0$ such that $|f(x)-f(y)| \leq \epsilon$ for all $x, y \in E$ such that $|x-y| \leq r(\epsilon)$. Let $a \in E$ be such that $|f(a)|>\epsilon$. Then $|f(x)|>\epsilon$ for every $x \in d_{E}(a, r(\epsilon))$, which proves that $W(f, \epsilon)$ is uniformly open in $E$.

Theorems 7 and 8 were proven in [10].

Theorem 7: Let $\mathcal{U}, \mathcal{V}$ be two ultrafilters on $E$ that are not contiguous. There exist uniformly open subsets $H \in \mathcal{U}, L \in \mathcal{V}$ of $E$ and $f \in \mathcal{D}(E)$ such that $f(x)=1$ for every $x \in H, f(x)=0$ for every $x \in L$.

Proof. Let $F \in \mathcal{U}, G \in \mathcal{V}$ be such that $\delta(F, G)=r>0$. Let $H=\bigcup_{b \in F} d_{E}\left(b, r^{-}\right)$. Then $F \cap G=\emptyset$.

Let $f$ be defined as $f(x)=1$ for every $x \in H$ and $f(x)=0$ for every $x \notin H$. Then $f(x)-f(y)=0$ for every $x, y \in H$ and $f(x)-f(y)=0$ for all $x, y \notin$ $H$. Now, take $x \in H$ and $y \notin H$. We have $\frac{f(x)-f(y)}{x-y}=\frac{1}{x-y}$ and hence $\left|\frac{f(x)-f(y)}{x-y}\right| \leq \frac{1}{r}$. Consequently, $\frac{f(x)-f(y)}{x-y}$ is bounded in $E$. Moreover, when $x$ and $y$ tend to a point $a \in E$, either both $x, y$ belong to $H$ or both $x, y$ do not belong to $H$, therefore $\frac{f(x)-f(y)}{x-y}=0$, so $f$ belongs to $\mathcal{D}(E)$. On the other hand, by construction, $H$ belongs to $\mathcal{U}$, and putting $L=E \backslash H$, we see that $G \subset L$, hence $L$ belongs to $\mathcal{V}$. Then, $f$ satisfies $f(x)=1$ for every $x \in H, f(x)=0$ for every $x \in L$. Moreover, by construction, we check that both $H$ and $L$ are uniformly open in $E$, which ends the proof.

Notation: We denote by $E^{\prime}$ another open subset of $\mathbb{K}$. Let $f$ be a mapping from $E$ to $E^{\prime}$ and let $\mathcal{U}$ be an ultrafilter on $E$. We denote by $\bar{f}(\mathcal{U})$ the ultrafilter admitting the basis $f(\mathcal{U})$.

Lemma M: Let $f$ be a uniformly continuous mapping from $E$ to $E^{\prime}$. Let $\mathcal{U}, \mathcal{V}$ be contiguous. Then $\bar{f}(\mathcal{U})$ and $\bar{f}(\mathcal{V})$ are contiguous. 
Proof. Set $\mathcal{U}^{\prime}=\bar{f}(\mathcal{U})$ and $\mathcal{V}^{\prime}=\bar{f}(\mathcal{V})$ and let $X \in \mathcal{U}^{\prime}, Y \in \mathcal{V}^{\prime}$. The familly of subsets $f^{-1}(f(\mathcal{U}))$ is obviously included in $\mathcal{U}$, hence the set $P=f^{-1}(X)$ belongs to $\mathcal{U}$ and similarly, the set $Q=f^{-1}(Y)$ belongs to $\mathcal{V}$. Now, we have $\delta(P, Q)=0$, so there exist a sequence $\left(a_{n}\right)_{n \in \mathbb{N}}$ of $P$ and a sequence $\left(b_{n}\right)_{n \in \mathbb{N}}$ of $Q$ such that $\lim _{n \rightarrow+\infty}\left(a_{n}-b_{n}\right)=0$. But since $f$ is uniformly continuous, in $E^{\prime}$ we have $\lim _{n \rightarrow+\infty} \delta^{\prime}\left(f\left(a_{n}\right), f\left(b_{n}\right)\right)=0$ and hence $\delta^{\prime}(X, Y)=0$ i.e. $\mathcal{U}^{\prime}(\mathcal{T}) \mathcal{V}^{\prime}$.

Notation: Given a uniformly continuous mapping $f$ from $E$ to $E^{\prime}$ and a class of contiguity $H$ on $E$, we will denote by $\bar{f}(H)$ the class of contiguity on $E^{\prime}:\{\bar{f}(\mathcal{U}) \mid \mathcal{U} \in$ $H\}$.

Given a filter $\mathcal{F}$ on $E$, we will denote by $\mathcal{I}(\mathcal{F})$ the ideal of the $f \in \mathcal{D}(E)$ such that $\lim _{\mathcal{F}} f(x)=0$. We will denote by $\mathcal{I}^{*}(\mathcal{F})$ the ideal of the $f \in \mathcal{D}(E)$ such that there exists a subset $L \in \mathcal{F}$ such that $f(x)=0$ for every $x \in L$. Given $a \in E$ we will denote by $\mathcal{I}(a)$ the ideal of the $f \in \mathcal{D}(E)$ such that $f(a)=0$.

We will denote by $\operatorname{Max}(\mathcal{D}(E))$ the set of maximal ideals of $\mathcal{D}(E)$ and by $\operatorname{Max}_{E}(\mathcal{D}(E))$ the set of maximal ideals of the form $\mathcal{I}(a), a \in E$.

The proof of Theorem 8 is easy and is not specific to the algebra $\mathcal{D}(E)[10]$.

Theorem 8: Given an ultrafilter $\mathcal{U}$ on $E, \mathcal{I}(\mathcal{U}), \mathcal{I}^{*}(\mathcal{U})$ are prime ideals of $\mathcal{D}(E)$.

Notation: We will denote by $|\cdot|_{\infty}$ the Archimedean absolute value of $\mathbb{R}$.

Theorem 9: Let $\mathcal{U}, \mathcal{V}$ be two ultrafilters on $E$. Then $\mathcal{I}(\mathcal{U})=\mathcal{I}(\mathcal{V})$ if and only if $\mathcal{U}$ and $\mathcal{V}$ are contiguous.

Proof. Suppose that $\mathcal{U}, \mathcal{V}$ are not contiguous. By Theorem 7 , there exist $H \in \mathcal{U}, L \in \mathcal{V}$ and $f \in \mathcal{D}(E)$ such that $f(x)=1$ for every $x \in H, f(x)=0$ for every $x \in L$. Consequently, $f$ belongs to $\mathcal{I}(\mathcal{U})$ but does not belong to $\mathcal{I}(\mathcal{V})$. Thus, $\mathcal{I}(\mathcal{F}) \neq \mathcal{I}(\mathcal{G})$.

Now, suppose that $\mathcal{U}, \mathcal{V}$ are contiguous. Let $f \in \mathcal{I}(\mathcal{U})$. Since $\mathcal{U}, \mathcal{V}$ are ultrafilters and since $f$ is bounded, $|f(x)|$ admits limits on both filters. Let $l=\lim _{\mathcal{V}}|f(x)|$, suppose $l>0$ and let $L \in \mathcal{V}$ be such that ||$f(x)|-l|_{\infty} \leq \frac{l}{3}$ for every $x \in L$, hence $|f(x)| \geq \frac{2 l}{3}$ for every $x \in L$. Let $H \in \mathcal{U}$ be such that $|f(x)| \leq \frac{l}{3}$ for all $x, y \in d_{E}(a, r), \stackrel{x}{\neq} \neq y . x \in H$. Since $f \in \mathcal{D}(E), f$ is uniformly continuous, hence there exists $\rho>0$ such that $\delta(x, y) \leq \rho$ implies $|f(x)-f(y)| \leq \frac{l}{4}$. And there exist $a \in H, b \in L$ such that $|a-b| \leq \rho$, hence $|f(a)-f(b)| \leq \frac{l}{4}$, a contradiction because $|f(a)| \leq \frac{l}{3}$ and $|f(b)| \geq \frac{2 l}{3}$.

Remark 5: As noticed in [10], Relation $(\mathcal{T})$ is not transitive in the case of the set of all filters on $E$. However, given a topological space $X$ satisfying the normality axiom, particularly, given a metric space $X$, then $(\mathcal{T})$ is transitive for ultrafilters and therefore is an equivalence relation on $U l(X)[10]$. 
Notation: We will denote by $Y_{(\mathcal{T})}(E)$ the set of equivalence classes on $U l(E)$ with respect to Relation $(\mathcal{T})$. Given $H \in Y_{(\mathcal{T})}(E)$, we will denote by $\mathcal{I}(H)$ the ideal $\mathcal{I}(\mathcal{U}), \mathcal{U} \in H$.

Let $f \in \mathcal{D}(E)$ and let $\epsilon$ be $>0$. We set $B(f, \epsilon)=\{x \in E|| f(x) \mid \leq \epsilon\}$.

Theorem 10 looks like certain Bezout-Corona statements [8], [17], [9], [10]. The proof is close to that given in [10] but here the functions in $\mathcal{D}(E)$ have more properties, allowing for a more specific proof.

Theorem 10: Let $f_{1}, \ldots, f_{q} \in \mathcal{D}(E)$ satisfying $\inf _{x \in E}\left(\max _{1 \leq j \leq q}\left|f_{j}(x)\right|\right)>0$. Then there exist $g_{1}, \ldots, g_{q} \in \mathcal{D}(E)$ such that $\sum_{j=1}^{q} f_{j}(x) g_{j}(x)=1$ for all $x \in E$.

Proof. Let $M=\inf _{x \in E}\left(\max _{1 \leq j \leq q}\left|f_{j}(x)\right|\right)$. Let $H_{j}=\left\{x \in E|| f_{j}(x) \mid \geq\right.$ $M\}, j=1, \ldots, q$ and let $F_{j}=\bigcup_{m=1}^{j} H_{m}, j=1, \ldots, q$. Let $g_{1}(x)=\frac{1}{f_{1}(x)}$ for all $x \in H_{1}$ and $g_{1}(x)=0$ for all $x \in E \backslash H_{1}$. In $H_{1}$, we have $\frac{g_{1}(x)-g_{1}(y)}{x-y}=\frac{f_{1}(y)-f_{1}(x)}{f_{1}(x) f_{1}(y)(x-y)}$, hence $\frac{g_{1}(x)-g_{1}(y)}{x-y}$ converges at each point $a \in H_{1}$ to $g_{1}^{\prime}(a)$.

Let us show that $g_{1}$ belongs to $\mathcal{D}(E)$. Let us fix $i \in I$ and let $h=\left\|f_{1}\right\|_{1}$. Let $x, y \in E_{i}$. If both $x, y$ belong to $E_{i} \backslash H_{1}$, we have $g_{1}(x)-g_{1}(y)=0$. If both $x, y$ belong to $H_{1}$, we have $g_{1}(x)-g_{1}(y)=\frac{f_{1}(y)-f_{1}(x)}{f_{1}(x) f_{1}(y)(x-y)}$ hence $\left|g_{1}(x)-g_{1}(y)\right| \leq \frac{\left\|f_{1}\right\|_{1}}{M^{2}}$.

Finally, suppose $x \in E_{1} \backslash H_{1}$ and $y \in H_{1}$. Now, we have $\left|g_{1}(x)-g_{1}(y)\right|=$ $\frac{1}{\mid f_{1}(y)} \mid \leq \frac{1}{M}$. On the other hand, by hypothesis, $\left|f_{1}(x)\right|<M,\left|f_{1}(y)\right| \geq M$, hence $\left|f_{1}(x)-f_{1}(y)\right| \geq M$, therefore $\frac{1}{|x-y|} \leq \frac{\left\|f_{1}\right\|_{1}}{\left|f_{1}(x)-f_{1}(y)\right|}$, hence

$$
\left|\frac{g_{1}(x)-g_{1}(y)}{x-y}\right| \leq \frac{\left\|f_{1}\right\|_{1}}{\left|f_{1}(y)\left(f_{1}(x)-f_{1}(y)\right)\right|} \leq \frac{\left\|f_{1}\right\|_{1}}{M^{2}} .
$$

Consequently, $g_{1}$ belongs to $\mathcal{D}(E)$.

Suppose now we have constructed $g_{1}, \ldots, g_{k} \in \mathcal{D}(E)$ satisfying $\sum_{j=1}^{k} f_{j} g_{j}(x)=1$ for all $x \in F_{k}$ and $\sum_{j=1}^{k} f_{j} g_{j}(x)=0$ for all $x \in E \backslash F_{k}$. Let $g_{k+1}$ be defined on $E$ by $g_{k+1}(x)=\frac{1}{f_{k+1}(x)}$ for every $x \in F_{k+1} \backslash F_{k}$ and $g_{k+1}(x)=0$ for every $x \in E \backslash\left(F_{k+1} \backslash F_{k}\right)$. In the same way as we did for $g_{1}$, we can check that $g_{k+1}$ belongs to $\mathcal{D}(E)$. 
Next, we have $\sum_{j=1}^{k+1} f_{j} g_{j}(x)=1$ for every $x \in F_{k+1}$ and $\sum_{j=1}^{k} f_{j} g_{j}(x)=0$ for every $x \in E \backslash F_{k+1}$. So, by an immediate recurrence, we can get functions $g_{1}, \ldots, g_{q} \in \mathcal{D}(E)$ such that

$\sum_{j=1}^{q} f_{j} g_{j}(x)=1$ for every $x \in E$, which ends the proof.

Corollary 10.1: Let $I$ be an ideal of $\mathcal{D}(E)$ different from $\mathcal{D}(E)$. The family $B(f, \epsilon), f \in I, \epsilon>0$, generates a filter $\mathcal{F}_{I}$ on $E$ such that $I \subset \mathcal{I}\left(\mathcal{F}_{I}\right)$.

\section{Main results}

Theorem 11: Let $\mathcal{M}$ be a maximal ideal of $\mathcal{D}(E)$. There exists an ultrafilter $\mathcal{U}$ on $E$ such that $\mathcal{M}=\mathcal{I}(\mathcal{U})$.

Proof. By Corollary 10.1, we have $\mathcal{M} \subset \mathcal{I}\left(\mathcal{F}_{\mathcal{M}}\right)$. Let $\mathcal{U}$ be an ultrafilter thinner than $\mathcal{F}_{\mathcal{M}}$. Then $\mathcal{I}\left(\mathcal{F}_{\mathcal{M}}\right) \subset \mathcal{I}(\mathcal{U})$, hence $\mathcal{M} \subset \mathcal{I}(\mathcal{U})$. But since $\mathcal{M}$ is a maximal ideal of $\mathcal{D}(E), \mathcal{M}$ is equal to $\mathcal{I}(\mathcal{U})$.

Corollary 11.1: For every maximal ideal $\mathcal{M}$ of $\mathcal{D}(E)$ there exists a unique $\mathcal{H} \in$ $Y_{(\mathcal{T})}(E)$ such that $\mathcal{M}=\mathcal{I}(\mathcal{U})$ for every $\mathcal{U} \in \mathcal{H}$.

Moreover, the mapping $\Psi$ that associates to each $\mathcal{M} \in \operatorname{Max}(\mathcal{D}(E))$ the unique $\mathcal{H} \in Y_{(\mathcal{T})}(E)$ such that $\mathcal{M}=\mathcal{I}(\mathcal{U})$ for every $\mathcal{U} \in \mathcal{H}$, is a bijection from $\operatorname{Max}(\mathcal{D}(E))$ onto $Y_{(\mathcal{T})}(E)$.

Theorem 12: $\quad$ Let $\mathcal{U}$ be an ultrafilter on $E$ such that, for every $f \in \mathcal{D}(E), f(x)$ converges on $\mathcal{U}$ in $\mathbb{K}$. Then $\mathcal{I}(\mathcal{U})$ is of codimension 1.

Proof. For each $f \in \mathcal{D}(E)$ we set $\chi(f)=\lim _{\mathcal{U}} f(x)$. Then $\mathcal{I}(\mathcal{U})$ is the ideal $\operatorname{Ker}(\chi)$ of $\mathcal{D}(E)$ and this is a maximal ideal because clearly $\chi(\mathcal{D}(E))=\mathbb{K}$. So, $\mathcal{I}(\mathcal{U})$ is a maximal ideal of $\mathcal{D}(E)$ of codimension 1 .

Corollary 12.1: $\quad$ Let $\mathcal{U}$ be a Cauchy ultrafilter on $E$. Then $\mathcal{I}(\mathcal{U})$ is of codimension 1 .

Proof. If $\mathcal{U}$ is convergent, there is nothing to prove. Suppose now that $\mathcal{U}$ does not converge. Since the functions of $\mathcal{D}(E)$ are uniformly continuous, they have a continuation to the completion $\widetilde{E}$ of $E$ and $\mathcal{U}$ defines an ultrafilter that converges in $\widetilde{E}$ to a point $a$. Given $f \in \mathcal{D}(E)$, let $\widetilde{f}$ be the continuation of $f$ in $\widetilde{E}$ : we have $\lim _{\mathcal{U}} f(x)=\widetilde{f}(a)$. So by Theorem $12, \mathcal{M}$ is of codimension 1 .

Corollary 12.2: $\quad$ Let $\mathbb{I K}$ be a locally compact field. Then every maximal ideal of $\mathcal{D}(E)$ is of codimension 1 . 
Proof. Let $\mathcal{M}$ be a maximal ideal of $\mathcal{D}(E)$. By Corollary 9.1 there exists an ultrafilter $\mathcal{U}$ such that $\mathcal{M}=\mathcal{I}(\mathcal{U})$. Let $f \in \mathcal{D}(E)$. Since $f$ is bounded, $f(E)$ is included in a compact subset $B$ of $\mathbb{K}$. Consequently, $f$ has a limit $\chi(f)$ along $\mathcal{U}$. Thus, the mapping $\chi$ from $\mathcal{D}(E)$ to $\mathbb{K}$ is a $\mathbb{K}$-algebra homomorphism and therefore $\mathcal{M}$ is of codimension 1.

We will now examine prime closed ideals of $\mathcal{D}(E)$.

Theorem 13: $\quad$ Let $\mathcal{U}$ be an ultrafilter on $E$ and let $\mathcal{P}$ be a prime ideal included in $\mathcal{I}(\mathcal{U})$. Let $L \in \mathcal{U}$ be uniformly open in $E$ and let $H=E \backslash L$. Let $u$ be the function defined on $E$ by $u(x)=1$ for every $x \in H, u(x)=0$ for every $x \in L$. Then $u$ belongs to $\mathcal{P}$.

Proof. By Theorem 7 we know that $u \in \mathcal{D}(E)$. By construction, $1-u$ does not belong to $\mathcal{I}(\mathcal{U})$ because $\lim _{\mathcal{U}} u(x)=1$. But $u(1-u)=0$, hence $u$ belongs to $\mathcal{P}$ because $\mathcal{P}$ is prime.

Corollary 13.1: Let $\mathcal{U}$ be an ultrafilter on $E$. The ideal of the $f \in \mathcal{D}(E)$ such that there exists a uniformly open subset $H \in \mathcal{U}$ of $E$ such that $f(x)=0$ for every $x \in H$ is included in every prime ideal $\mathcal{P} \subset \mathcal{I}(\mathcal{U})$.

Theorem 14: The closure of a prime ideal of $\mathcal{D}(E)$ is a maximal ideal.

Proof. The proof is similar to Theorem 12 in [10]. Let $\mathcal{P}$ be a prime ideal of $\mathcal{D}(E)$ included in a maximal ideal $\mathcal{M}=\mathcal{I}(\mathcal{U})$. Let $f$ belong to $\mathcal{I}(\mathcal{U})$. Let us take $\epsilon>0$ and let us find $h \in \mathcal{P}$ such that $\|f-h\| \leq \epsilon$. By Theorem 7 , we can find a uniformly open subset $L \in \mathcal{U}$ of $E$ such that $|f(x)| \leq \epsilon$ for every $x \in L$. Let $u$ be the characteristic function of $E \backslash L$. By Theorem 13, $u$ belongs to $\mathcal{P}$ and hence so does $u f$. We then check that $\|f-u f\| \leq \epsilon$. Thus, $\mathcal{P}$ is dense in $\mathcal{M}$.

Corollary 14.1: $\quad$ Let $\mathcal{P}$ be a prime ideal of $\mathcal{D}(E)$. There exists a unique maximal ideal $\mathcal{M}$ of $\mathcal{D}(E)$ containing $\mathcal{P}$.

Corollary 14.2: $\quad$ Every prime closed ideal of $\mathcal{D}(E)$ is a maximal ideal.

Let us recall the main definitions concerning multiplicative semi-norms [3], [4], [5], [8], [10], [13], [14].

Notation and definition: We denote by $\operatorname{Mult}(\mathcal{D}(E), \|$. $\|)$ the set of multiplicative semi-norms of $\mathcal{D}(E)$ provided with the topology of pointwise convergence. Given $\phi \in \operatorname{Mult}(\mathcal{D}(E), \|$. $\|)$, the set of the $f \in \mathcal{D}(E)$ such that $\phi(f)=0$ is a closed prime ideal called the kernel of $\phi$. It is denoted by $\operatorname{Ker}(\phi)$.

We denote by Mult $_{m}(\mathcal{D}(E),\|\|$.$) the set of multiplicative semi-norms of \mathcal{D}(E)$ whose kernel is a maximal ideal and by $\operatorname{Mult}_{1}(\mathcal{D}(E), \|$. $\|)$ the set of multiplicative semi-norms of $\mathcal{D}(E)$ whose kernel is a maximal ideal of codimension 1.

Let $a \in E$. The mapping $\varphi_{a}$ from $\mathcal{D}(E)$ to $\mathbb{R}$ defined by $\varphi_{a}(f)=|f(a)|$ belongs to $\operatorname{Mult}(\mathcal{D}(E),\|\|$.$) . Let \mathcal{U}$ be an ultrafilter on $E$. By Urysohn's Theorem, given $f \in \mathcal{D}(E)$, the mapping from $E$ to $\mathbb{R}$ that sends $x$ to $|f(x)|$ admits a limit along $\mathcal{U}$. We set $\varphi_{\mathcal{U}}(f)=\lim _{\mathcal{U}}|f(x)|$. Moreover, we denote by $\operatorname{Mult}_{E}(\mathcal{D}(E),\|\|$.$) the$ 
set of multiplicative semi-norms of $\mathcal{D}(E)$ of the form $\varphi_{a}, a \in E$. Consequently, by definition, $\operatorname{Mult}_{E}(\mathcal{D}(E),\|\cdot\|)$ is a subset of $\operatorname{Mult}_{1}(\mathcal{D}(E),\|\cdot\|)$.

The following Theorems 15 and 16 are immediate and well known:

Theorem 15: Let $a \in E$. Then $\mathcal{I}(a)$ is a maximal ideal of $\mathcal{D}(E)$ of codimension 1 and $\varphi_{a}$ belongs to $\operatorname{Mult}_{1}(\mathcal{D}(E), \|$. $\|)$.

Corollary 15.1: $\operatorname{Mult}_{E}(\mathcal{D}(E),\|\cdot\|)$ is included in $\operatorname{Mult}_{1}(\mathcal{D}(E),\|\cdot\|)$.

Theorem 16: Let $\mathcal{U}$ be an ultrafilter on $E$. Then $\varphi_{\mathcal{U}}$ belongs to the closure of $\operatorname{Mult}_{E}(\mathcal{D}(E),\|\cdot\|)$.

Now, Corollary 16.1 is an immediate consequence of Theorems 14, 15 and 16 and Corollary 12.1:

Corollary 16.1 : $\operatorname{Mult}(\mathcal{D}(E),\|\cdot\|)=\operatorname{Mult}_{m}(\mathcal{D}(E),\|\cdot\|)$. Furthermore, if $\mathbb{K}$ is locally compact then $\operatorname{Mult}(\mathcal{D}(E),\|\cdot\|)=\operatorname{Mult}_{1}(\mathcal{D}(E),\|\cdot\|)$.

Remark 6: Suppose $\mathbb{K}$ is locally compact and $E$ is a disk in an algebraically closed complete ultrametric field. There do exist ultrafilters on $E$ that do not converge. Let $\mathcal{U}$ be such an ultrafilter. Then $\varphi_{\mathcal{U}}$ belongs to $M$ ult $t_{1}(\mathcal{D}(E),\|\|$.$) but$ does not belong to $\operatorname{Mult}_{E}(\mathcal{D}(E),\|\cdot\|)$.

Remark 7: In $\mathcal{H} \in Y_{(\mathcal{T})}(E)$ the various ultrafilters $\mathcal{U} \in \mathcal{H} \in Y_{(\mathcal{T})}(E)$ define various prime ideals of $\mathcal{D}(E)$ and it is not clear whether these ideals are minimal among the set of prime ideals of $\mathcal{D}(E)$.

Theorem 17: The topology induced on $E$ by the one of $\operatorname{Mult}_{E}(\mathcal{D}(E),\|\cdot\|)$ is equivalent to the metric topology induced on $E$ by the field $\mathbb{K}$.

Proof. The proof made for Theorem 15 in [10] holds with no change. Let us recall it. Let $a \in E$. The filter of neighborhoods of $a$ admits for basis the family of disks $d_{E}\left(a, r^{-}\right), r>0$. But we can check that such a disk is induced by a neighborhood of $\varphi_{a}$ with respect to the topology of $M u l t_{E}(\mathcal{D}(E),\|\cdot\|)$ and to the metric topology of $E$. Given $\varphi_{a} \in \operatorname{Mult}_{E}(\mathcal{D}(E),\|\cdot\|)$, we set

$W^{\prime}\left(\varphi_{a}, f_{1}, \ldots, f_{q}, \epsilon\right)=W\left(\varphi_{a}, f_{1}, \ldots, f_{q}, \epsilon\right) \cap \operatorname{Mult}_{E}(\mathcal{D}(E), \|$. \|). Let $r \in] 0,1[$. There exist $u \in \mathcal{D}(E)$ such that $u(x)=0$ for every $x \in d_{E}\left(a, r^{-}\right)$and $u(x)=1$ for every $x \in E \backslash d_{E}\left(a, r^{-}\right)$. Consequently, $W^{\prime}\left(\varphi_{a}, u, r\right)$ is the set of the $\varphi_{b}$ such that $|b-a| \leq r$. Hence the topology of $\operatorname{Mult}_{E}(\mathcal{D}(E),\|\|$.$) is thinner or equal to the$ metric topology of $E$. Now, since each $f_{j}$ is continuous, the set of the $x \in E$ such that ||$f_{j}(x)|-| f_{j}(a)||_{\infty} \leq \epsilon$ for every $j=1, \ldots, q$ is open and hence contains a ball $d_{E}\left(a, r^{-}\right)$of $E$. Consequently, the topology of $E$ is thinner or equal to this of $\operatorname{Mult}_{E}(\mathcal{D}(E),\|\cdot\|)$, which finishes the proof that the topology induced on $E$ by $\operatorname{Mult}(\mathcal{D}(E),\|\cdot\|)$ coincides with the metric topology of $E$.

Theorem 18 was proven in [19] for the algebra of bounded continuous functions. In [10] we proved it again for algebras of bounded continuous functions and for algebras of bounded uniformly continuous functions, in a different way. Here we adapt that proof to the algebra $\mathcal{D}(E)$. 
Theorem 18: Let $\mathcal{M}$ be a maximal ideal of $\mathcal{D}(E)$. Let $T$ be the field $\frac{\mathcal{D}(E)}{\mathcal{M}}$ and let $\theta$ be the canonical surjection from $\mathcal{D}(E)$ onto $T$. Given any ultrafilter $\mathcal{U}$ such that $\mathcal{I}(\mathcal{U})=\mathcal{M}$, the quotient norm $\|$. $\|^{\prime}$ on $T$ is defined by $\|\theta(f)\|^{\prime}=\lim _{\mathcal{U}}|f(s)|$ and hence is multiplicative.

Proof. Let $t \in T$ and let $f \in \mathcal{D}(E)$ be such that $\theta(f)=t$. Let $\mathcal{U}$ be an ultrafilter such that $\mathcal{I}(\mathcal{U})=\mathcal{M}$. So, on $T$ we have an absolute value $\psi$ defined as $\psi(t)=\lim _{\mathcal{U}}|f(s)|$. That absolute value is continuous with respect to the quotient norm $\|$. $\|^{\prime}$ of $T$. Indeed, by construction, we have $\|f\| \geq \lim _{\mathcal{U}}|f(s)|=\psi(t)$. But $\|t\|^{\prime}=\inf _{h \in \mathcal{M}}\|f+h\|$ and of course, $\psi(\theta(t+h))=\psi(t)=\lim _{\mathcal{U}}|f(s)|$. Consequently $\|t\|^{\prime} \geq \psi(t)$.

Conversely, let $V \in \mathcal{U}$ be such that $|f(x)| \leq \lim _{\mathcal{U}}|f(s)|+\epsilon$ for every $x \in$ $V$. There exist $f_{1}, \ldots, f_{q} \in \mathcal{M}$ and $\epsilon>0$ sucht that $\bigcap_{j=1}^{q} B\left(f_{j}, \epsilon\right) \subset V$. Let $X=$ $\bigcap_{j=1}^{q} B\left(f_{j}, \epsilon\right)$. By Theorem 6 , since $f_{j} \in \mathcal{D}(E)$ for every $j=1, \ldots, q, X$ is uniformly open in $E$. Consequently, by Theorem 5 there exists $u \in \mathcal{D}(E)$ such that $u(x)=0$ for every $x \in X, u(x)=1$ for every $x \in E \backslash X$. Then $u(1-u)=0$. But $1-u \notin \mathcal{M}$. Hence, $u$ belongs to $\mathcal{M}$ and then $\theta(f-u f)=\theta(f)=t$. But by construction, $(f-u f)(x)=0$ for every $x \in E \backslash X$ and $(f-u f)(x)=f(x)$ for every $x \in X$. Consequently, $\|f-u f\| \leq \lim _{\mathcal{U}}|f(s)|+\epsilon$ and therefore $\|t\|^{\prime}=\|\theta(f-u h)\| \leq$ $\lim _{\mathcal{U}}|f(s)|+\epsilon$. This finishes the proof that the equality $\|\theta(f)\|^{\prime}=\lim _{\mathcal{U}}|f(s)|$. Now, such a norm defined as $\|\theta(f)\|^{\prime}=\lim _{\mathcal{U}}|f(s)|$ is obviously multiplicative.

Remark 8: A similar proof is given in [10] for Theorem 16 where we forgot to mention the use of a lemma such as Theorem 6 in the case of algebra $B$.

Definition: Recall that given a commutative Banach $\mathbb{K}$-algebra $T$ with unity, every maximal ideal of $T$ is the kernel of at least one continuous multiplicative semi-norm [2], [3]. The algebra $T$ is said to be multbijective if every maximal ideal is the kernel of only one continuous multiplicative semi-norm.

Remark 9: There exist ultrametric Banach $\mathbb{K}$-algebras that are not multbijective $[1],[2],[3]$.

Theorem 19: $\mathcal{D}(E)$ is multbijective.

Proof. Let $\mathcal{M}$ be a maximal ideal of $\mathcal{D}(E)$ and let $T$ be the field $\frac{\mathcal{D}(E)}{\mathcal{M}}$. By Theorem 18, the quotient norm of $T$ is multiplicative. But then, $T$ admits only one continuous multiplicative semi-norm: its quotient $\mathbb{K}$-algebra norm. Consequently, $\mathcal{D}(E)$ admits only one continuous multiplicative semi-norm whose kernel is $\mathcal{M}$, which proves that $\mathcal{D}(E)$ is multbijective. 
By Theorem 19 and Corollary 14.2, we can now state Corollary 19.1:

Corollary 19.1: The mapping that associates to each $\phi \in \operatorname{Mult}(\mathcal{D}(E), \|$. $\|)$ its kernel $\operatorname{Ker}(\phi)$ is a bijection from $\operatorname{Mult}(\mathcal{D}(E), \|$. \|) onto $\operatorname{Max}(\mathcal{D}(E))$.

By Theorem 11, Corollary 11.1 and Theorem 19, we have Corollary 19.2:

Corollary 19.2: For every $\phi \in \operatorname{Mult}(\mathcal{D}(E), \|$. $\|)$ there exists a unique $\mathcal{H} \in$ $Y_{(\mathcal{T})}(E)$ such that $\phi(f)=\lim _{\mathcal{U}}|f(x)|$ for every $f \in \mathcal{D}(E)$, for every $\mathcal{U} \in \mathcal{H}$.

Moreover, the mapping $\widetilde{\Psi}$ that associates to each $\phi \in \operatorname{Mult}(\mathcal{D}(E), \|$. $\|)$ the unique $\mathcal{H} \in Y_{(\mathcal{T})}(E)$ such that $\phi(f)=\lim _{\mathcal{U}}|f(x)|$ for every $f \in \mathcal{D}(E)$, for every $\mathcal{U} \in \mathcal{H}$, is a bijection from $\operatorname{Mult}(\mathcal{D}(E), \|$. $\|)$ onto $Y_{(\mathcal{T})}(E)$.

Now, by Theorems 15 and 16, we have Corollary 19.3:

Corollary 19.3: $\operatorname{Mult}_{E}(\mathcal{D}(E), \|$. $\|)$ is dense in $\operatorname{Mult}(\mathcal{D}(E), \|$. \|).

Proof. Indeed, let $\psi \in \operatorname{Mult}(\mathcal{D}(E), \|$. $\|)$. By Theorem 16, $\operatorname{Ker}(\psi)$ is a maximal ideal $\mathcal{I}(\mathcal{U})$ with $\mathcal{U}$ an ultrafilter on $E$; but then by Theorem $19, \psi=\varphi_{\mathcal{U}}$ and hence $\psi$ belongs to the closure of $\operatorname{Mult}_{E}(\mathcal{D}(E),\|\|$.$) .$

Theorem 20: For every $\phi \in \operatorname{Mult}(\mathcal{D}(E), \|$. $\|)$, $\phi$ satisfies $\phi(f) \leq\|f\|_{0} \quad$ for every $f \in \mathcal{D}(E)$.

Proof. By Theorem 19, $\mathcal{D}(E)$ is multbijective, then by Theorem 11, every element of $\operatorname{Mult}_{m}(\mathcal{D}(E),\|\|$.$) is of the form \varphi_{\mathcal{U}}$ where $\mathcal{U}$ is an ultrafilter on $E$. Of course, we have $\varphi_{\mathcal{U}}(f) \leq\|f\|_{0}$ for every $f \in \mathcal{D}(E)$ and consequently, $\phi(f) \leq\|f\|_{0}$ for every $f \in \mathcal{D}(E)$, for every $\phi \in \operatorname{Mult}_{m}(\mathcal{D}(E), \|$. \|). But by Corollary 16.1, Mult $_{m}(\mathcal{D}(E), \|$. $\|)=\operatorname{Mult}(\mathcal{D}(E), \|$. $\|)$. Thererfore $\phi(f) \leq\|f\|_{0}$ for every $f \in \mathcal{D}(E)$, for every $\phi \in \operatorname{Mult}(\mathcal{D}(E), \|$. $\|)$.

Notation: On $\mathcal{D}(E)$ we denote by $\|\cdot\|_{s i}$ the semi-norm of $\mathcal{D}(E)$ defined as $\|f\|_{s i}=$ $\lim _{n \rightarrow+\infty} \sqrt[n]{\left\|f^{n}\right\|}$

Le us recall Proposition F (See for instance Theorem 6.19 in [3]):

Proposition F: Let $T$ be a normed $\mathbb{K}$-algebra whose norm is $\|$. $\|$. Then $\|$. $\|_{s i}$ is a power multiplicative semi-norm on $T$ such that $\|f\|_{s i}=\sup \{\varphi(f) \mid \varphi \in$ $\operatorname{Mult}(\mathcal{D}(E), \|$. $\|\}$.

Then we can state Theorem 21:

Theorem 21: $\|f\|_{s i}=\|f\|_{0}$ for every $f \in \mathcal{D}(E)$.

Proof. We have $\|f\|_{s i}=\sup \{\varphi(f) \mid \varphi \in \operatorname{Mult}(\mathcal{D}(E), \|$. $\|\}$. But by Corollary 16.1, we have $\operatorname{Mult}(\mathcal{D}(E), \|$. $\|)=\operatorname{Mult}_{m}(\mathcal{D}(E), \|$. $\|)$, hence $\|f\|_{s i}=$ $\sup \left\{\varphi(f) \mid \varphi \in \operatorname{Mult}_{m}(\mathcal{D}(E), \|\right.$. $\|\}$. Consequently, $\|f\|_{s i} \geq|f(x)|$ for every $x \in E$, for every $f \in \mathcal{D}(E)$ and hence $\|f\|_{s i} \geq\|f\|_{0}$ for every $f \in \mathcal{D}(E)$. On the other hand, by Theorem 20, we have $\phi(f) \leq\|f\|_{0}$ for every $f \in \mathcal{D}(E)$, for every $\phi \in \operatorname{Mult}(\mathcal{D}(E), \|$. $\|)$, hence $\|f\|_{s i} \leq\|f\|_{0}$ for every $f \in \mathcal{D}(E)$ and finally $\|f\|_{s i}=\|f\|_{0}$ for every $f \in \mathcal{D}(E)$. 
Theorem 22: Suppose $\mathbb{K}$ is algebraically closed. Let $\mathcal{U}$ be an ultrafilter on $\mathbb{K}$ and suppose there exists $P \in \mathbb{K}[x], P \neq 0$ satisfying $\lim _{\mathcal{U}} P(x)=0$. Then $\mathcal{U}$ is a principal ultrafilter.

Proof. Let $P(x)=\prod_{j=1}^{q}\left(x-a_{j}\right)$. Let $\mathcal{F}$ be the filter admitting for basis the family of sets $\Lambda(r)=\bigcup_{j=1}^{q} d\left(a_{j}, r\right), r>0$. Suppose first that $\mathcal{U}$ is not secant with $\mathcal{F}$. There exist $\rho>0$ and $H \in \mathcal{U}$ such that $\Lambda(\rho) \cap H=\emptyset$. Then $|P(x)| \geq \rho^{q}$ for every $x \in H$, a contradiction to the hypothesis $\lim _{\mathcal{U}} P(x)=0$. Consequently, $\mathcal{U}$ is secant with $\mathcal{F}$. Hence it is obviously secant with the filter of neighborhoods of one of the points $a_{j}$ and therefore, it converges to this point.

As a consequence, we have Theorem 23:

Theorem 23: Suppose that $\mathbb{K}$ is algebraically closed and that $E$ is a closed bounded subset of $\mathbb{K}$ with infinitely many points and let $\mathcal{M}$ be a maximal ideal of $\mathcal{D}(E)$ of the form $\mathcal{I}(\mathcal{U})$ where $\mathcal{U}$ is not principal. Then $\mathcal{M}$ is of infinite codimension.

Proof. Indeed, by Theorem 22 the ideal $\mathcal{M}$ contains no polynomials different from 0 , hence $\frac{\mathcal{D}(E)}{\mathcal{M}}$ contains a subfield isomorphic to $\mathbb{K}(x)$ and therefore is not of finite codimension.

Remark 10: Suppose $\mathbb{I K}$ is algebraically closed and let $E=\mathbb{K}$. Then the algebra $\mathcal{D}(E)$ contains no polynomial. In such a case, it is not clear whether all maximal ideals not defined by points of $\mathbb{K}$ are of infinite codimension.

Remark 11: Concerning uniformly continuous functions, it has been shown in [10] that two ultrafilters that are not contiguous define two distinct continuous multiplicative semi-norms. By Corollary 11.1 we know that the same holds in $\mathcal{D}(E)$.

Now, concerning bounded analytic functions inside the disk $F=\{x \in \mathbb{K}|x|<$ $1\}$, in [7], it was shown that the same property holds for a large set of ultrafilters on $F$. However, the question remains whether it holds for all ultrafilters on $F$.

The following lemma is well known and comes from the fact that on a vector space of finite dimension over a complete field, two norms are equivalent.

Lemma $\mathbf{U}: \quad$ Let $E$ be an algebraic extension of $\mathbb{K}$ of degree $t$ of the form $\mathbb{K}[a]$ provided with the absolute value $\mid$. | which expands that of $\mathbb{K}$. Given $x \in E$, let $x=\sum_{j=0}^{t-1} a^{j} x_{j}$ and let $\|x\|=\max _{0 \leq j \leq t-1}\left|x_{j}\right|$. Then the norm $\|$. $\|$ is equivalent to the absolute value |. |.

Theorem 24 : Let $E$ be an algebraic extension of $\mathbb{I K}$ of degree $t$ of the form $\mathbb{K}[a]$ provided with the absolute value which expands that of $\mathbb{K}$. Let $f$ be a strictly 
differentiable function from $E$ to $E$. There exists $f_{0}, \ldots, f_{t-1} \in \mathcal{D}(E)$ such that $f=\sum_{j=0}^{t-1} a^{j} f_{j}$

Proof. For every $x \in E$, we can write $f(x)=\sum_{j=0}^{t-1} a^{j} f_{j}(x)$ with $f_{j}(x) \in \mathbb{K}, 0 \leq$ $j \leq t-1$. We will show that each function $f_{j}$ belongs to $\mathcal{D}(E)$.

By definition, given any $a \in E, \frac{f(x)-f(y)}{x-y}$ has a limit $f^{\prime}(a) \in \mathrm{E}$ when $x$ and $y$ tend to $a$ while $x \neq y$. Let us write $f^{\prime}(a)=\sum_{j=0}^{t-1} a^{j} l_{j}$, hence

$$
\frac{f(x)-f(y)}{x-y}-f^{\prime}(a)=\sum_{j=0}^{t-1} a^{j}\left(\frac{f_{j}(x)-f_{j}(y)}{x-y}-l_{j}\right) .
$$

Since $\lim _{\substack{x \rightarrow a, y \rightarrow a \\ x \neq y}} \frac{f(x)-f(y)}{x-y}-f^{\prime}(a)=0$, by Lemma U, we can see that for each $j=0, \ldots, t-1$, we have $\lim _{\substack{x \rightarrow a, y \rightarrow a \\ x \neq y}} \frac{f_{j}(x)-f_{j}(y)}{x-y}-l_{j}=0$. Consequently, $f_{j}$ does belong to $\mathcal{D}(E)$ and that ends the proof of Theorem 24 .

We can now prove Theorem 25 that will let us show Theorem 26:

Theorem 25: $\quad$ Let $E$ be a finite algebraic extension of $\mathbb{I K}$ provided with the absolute value which expands that of $\mathbb{K}$. Suppose there exists a morphism of $\mathbb{K}$-algebra, $\chi$, from $\mathcal{D}(E)$ onto $E$. Let $\widehat{\mathcal{D}}(E)$ be the E-algebra of strictly differentiable functions from $E$ to $E$. Then $\chi$ has continuation to a morphism of E-algebra $\widehat{\chi}$ from $\widehat{\mathcal{D}}(E)$ to $E$.

Proof. The proof is similar to the proof of Proposition 3.5 in [10]. Let $t=$ $[\mathrm{E}: \mathbb{K}]$. By the primitive element theorem, there exists $a \in \mathrm{E}$ such that $\mathrm{E}=\mathbb{K}[a]$. Let $f, g \in \widehat{\mathcal{D}}(E)$. Then $f$ is of the form $\sum_{j=0}^{t-1} a^{j} f_{j}, f_{j} \in \mathcal{D}(E), j=0, \ldots, d-1$ and $g$ is of the form $\sum_{j=0}^{t-1} a^{j} g_{j}, g_{j} \in \mathcal{D}(E), j=0, \ldots, t-1$. By Theorem 24, all the $f_{j}$ and the $g_{j}$ belong to $\mathcal{D}(E)$.

Now, we can define $\widehat{\chi}$ on $\widehat{\mathcal{D}}(E)$ as $\widehat{\chi}(f)=\sum_{j=0}^{t-1} a^{j} \chi\left(f_{j}\right)$. Then obviously, $\widehat{\chi}$ is $\mathbb{K}$-linear. On the other hand, for each $q \in \mathbb{N}, a^{q}$ is of the form $P_{q}(a)$ where $P_{q} \in \mathbb{K}[x], \operatorname{deg}\left(P_{q}\right) \leq t-1$. Then $\widehat{\chi}\left(a^{q}\right)=\widehat{\chi}\left(P_{q}(a)\right)=P_{q}(\widehat{\chi}(a))=P_{q}(a)=a^{q}$. Next,

$$
\widehat{\chi}(f g)=\widehat{\chi}\left(\left(\sum_{j=0}^{t-1} a^{j} f_{j}\right)\left(\sum_{j=0}^{t-1} a^{j} g_{j}\right)\right)=\widehat{\chi}\left(\sum_{\substack{0 \leq m \leq t-1 \\ 0 \leq n \leq t-1}} a^{m+n} f_{m} g_{n}\right)
$$




$$
=\sum_{\substack{0 \leq m \leq t-1 \\ 0 \leq n \leq t-1}} a^{m+n} \chi\left(f_{m}\right) \chi\left(g_{n}\right)=\left(\sum_{j=0}^{t-1} a^{j} \chi\left(f_{j}\right)\right)\left(\sum_{j=0}^{t-1} a^{j} \chi\left(g_{j}\right)\right)=\chi(f) \chi(g) .
$$

That finishes the proof that $\chi$ is a E-algebra morphism from $\widehat{\mathcal{D}}(E)$ to $\mathrm{E}$.

Theorem 26: Every maximal ideal of finite codimension of $\mathcal{D}(E)$ is of codimension 1.

Proof. The proof follows from Theorem 3.7 in [10]. Let $\mathcal{M}$ be a maximal ideal of finite codimension of $\mathcal{D}(E)$, let $\mathrm{E}$ be the field $\frac{\mathcal{D}(E)}{\mathcal{M}}$ and let $\chi$ be the canonical surjective morphism from $\mathcal{D}(E)$ over $\mathrm{L}$. Since $\mathcal{M}$ is of finite codimension, $\mathrm{E}$ is a finite extension of $\mathbb{K}$ and hence has a unique absolute value expanding that of $\mathbb{K}$. Consequently, $\mathrm{E}$ is complete for this absolute value. Let $\widehat{\mathcal{D}}(E)$ be the $\mathrm{E}$-algebra of strictly differentiable functions from $E$ to $\mathrm{E}$. By Theorem $25, \chi$ has continuation to a Ł-algebra morphism $\widehat{\chi}$ from $\widehat{\mathcal{D}}(E)$ to $\mathrm{L}$.

Let $f \in \mathcal{D}(E)$. Then $\chi(f)$ is an element $b$ of $\mathrm{E}$ and of course, $f-b \in \widehat{\mathcal{D}}(E)$. Now, by Theorem 25, $\widehat{\chi}$ is surjective on $\mathrm{E}$, hence $\operatorname{Ker}(\widehat{\chi})$ is a maximal ideal $\widehat{\mathcal{M}}$ of $\widehat{\mathcal{D}}(E)$. Then by Theorem 11, there exists an ultrafilter $\mathcal{U}$ on $E$ such that $\widehat{\mathcal{M}}$ is the ideal of the $g \in \widehat{A}$ such that $\lim _{\mathcal{U}} g(x)=0$. So, we have $\lim _{\mathcal{U}}(f(x)-b)=0$, therefore $\lim _{\mathcal{U}}(f(x))=b$. But since $\mathbb{K}$ is complete, this proves that $b$ belongs to $\mathbb{K}$ and therefore $\chi(\mathcal{D}(E))=\mathbb{K}$ i.e. $\mathrm{E}=\mathbb{K}$.

By Theorem 26 and Corollary 12.1, we can state this corollary:

Corollary 26.1: $\quad$ Let $\mathcal{U}$ be an ultrafilter on $E$. The following 3 statements are equivalent:

i) $\mathcal{I}(\mathcal{U})$ is of codimension 1 ,

ii) $\mathcal{I}(\mathcal{U})$ is of finite codimension,

iii) for every $f \in \mathcal{D}(E)$, the filter generated by $f(\mathcal{U})$ converges in $\mathbb{K}$.

Corollary 26.2: $\quad$ Every maximal ideal of $\mathcal{D}(E)$ of finite codimension is of codimension 1.

The following Theorem is almost classical [2], [3]:

Theorem 27: $\quad$ Suppose $\mathbb{K}$ is not locally compact. There exists a sequence $\left(b_{n}\right)_{n \in \mathbb{N}}$ in $\mathbb{K}$ such that either the sequence $\left|b_{n}-b_{n+1}\right|$ is a strictly monotonous, of limit $r \in] 0,+\infty\left[\right.$, or $\left|b_{n}-b_{m}\right|$ is a constant whenever $m \neq n \in \mathbb{N}$.

Proof. Indeed, since $\mathbb{K}$ is not locally compact, either the set $|\mathbb{K}|=\{|x| \mid x \in$ $\mathbb{K}\}$ is not discrete and hence is dense in $[0,+\infty[$ or the residue field of $\mathbb{I K}$ is infinite. If $|\mathbb{K}|$ is dense in $\left[0,+\infty\left[\right.\right.$, there exists a sequence $\left(b_{n}\right)_{n \in \mathbb{N}}$ such that $\left|b_{n}-b_{n+1}\right|$ is strictly monotonous, of limit $r \in] 0,+\infty[$. And if the residue field of $\mathbb{K}$ is infinite, then there exists sequences $\left(b_{n}\right)_{n \in \mathbb{N}}$ such that $\left|b_{n}-b_{m}\right|=c$ for every $n \neq m$. 
Theorem 28: $\quad$ Suppose that $\mathbb{K}$ is not locally compact and that $E$ contains a sequence $\left(a_{n}\right)_{n \in \mathbb{N}}$ such that $\left|a_{n}-a_{m}\right| \geq r>$ for all $m \neq n$. Let $\left(b_{n}\right)_{n \in \mathbb{N}}$ be a sequence of $\mathbb{I K}$ such that either the sequence $\left|b_{n}-b_{n+1}\right|$ is a strictly monotonous, of limit $r \in] 0,+\infty\left[\right.$, or $\left|b_{n}-b_{m}\right|$ is a constant whenever $m \neq n \in \mathbb{N}$.

Then, there exists an ultrafilter $\mathcal{U}$ on $E$ thinner than the sequence $\left(a_{n}\right)_{n \in \mathbb{N}}$ and $f \in \mathcal{D}(E)$ such that the filter generated by $f(\mathcal{U})$ is thinner than the sequence $\left(b_{n}\right)_{n \in \mathbb{N}}$ and does not converge.

Proof. By hypothesis, there exists $\lambda>0$ such that $\left|b_{m}-b_{n}\right| \geq \lambda$ for all $m \neq n$. Now, we can define a function $f$ from $E$ to $\mathbb{K}$ as $f(x)=b_{n}$ for every $x \in d_{E}\left(a_{n}, r^{-}\right)$and (for instance) $f(x)=0$ for every $x \in E \backslash\left(\bigcup_{n \in \mathbb{N}} d_{E}\left(a_{n}, r^{-}\right)\right)$. We can check that $f$ belongs to $\mathcal{D}(E)$.

Now, let $\mathcal{U}$ be an ultrafilter on $E$ thinner than the sequence $\left(a_{n}\right)_{n \in \mathbb{N}}$. Then, the filter $\mathcal{W}$ generated by $f(\mathcal{U})$ on $\mathbb{K}$ is thinner than the sequence $\left(b_{n}\right)_{n \in \mathbb{N}}$. Every element $X$ of $\mathcal{W}$ contains several terms of the sequence $\left(b_{n}\right)_{n \in \mathbb{N}}$, for instance $b_{m}, b_{n}$ with $m \neq n$, hence $\operatorname{diam}(X) \geq \lambda$ and therefore $\mathcal{W}$ does not converge.

Theorem 29: $\quad$ The algebra $\mathcal{D}(E)$ admits maximal ideals of infinite codimension if and only if $\mathrm{IK}$ is not locally compact.

PROOF. If $\mathbb{I K}$ is locally compact, then by Theorem 12 , every maximal ideal of $\mathcal{D}(E)$ is of codimension 1 .

Now, suppose that $\mathbb{K}$ is not locally compact. By Theorem 27 there exists a sequence $\left(b_{n}\right)_{n \in \mathbb{N}}$ in $\mathbb{K}$ such that either the sequence $\left|b_{n}-b_{n+1}\right|$ is a strictly monotonous, of limit $r \in] 0,+\infty\left[\right.$, or $\left|b_{n}-b_{m}\right|$ is a constant whenever $m \neq n \in \mathbb{N}$. So, by Theorem 28 in both cases there exists an ultrafilter $\mathcal{U}$ on $E$ and $f \in B$ such that the filter generated by $f(\mathcal{U})$ is thinner than the sequence $\left(b_{n}\right)_{n \in \mathbb{N}}$ and does not converge. Consequently, $\mathcal{I}(\mathcal{U})$ is a maximal ideal of $\mathcal{D}(E)$ of infinite codimension.

Notation: Given $r \in] 0,+\infty\left[\right.$, and $a \in E$, we denote by $d_{E}\left(a, r^{-}\right)$the ball of $E$ $\{x \in E:|x-a|<r\}$ and we denote by card $(E, r)$ the cardinal of the set of disks $d_{E}\left(a, r^{-}\right), a \in E$. We denote by $U$ the disk $d(0,1)$ of $\mathbb{K}$.

We can now add some precisions on maximal ideals of infinite codimension by making certain hypotheses on $E$ and $\mathbb{I K}$.

Theorem 30: $\quad$ Suppose that we have $\operatorname{card}(E, r) \leq \operatorname{card}(U, r)$ for every $r \in] 0,1]$. Let $\mathcal{U}$ be an ultrafilter on $E$. Either $\mathcal{U}$ is convergent and then $\mathcal{I}(\mathcal{U})$ is of codimension 1 , or $\mathcal{U}$ is not convergent and then $\mathcal{I}(\mathcal{U})$ is of infinite codimension.

ProOF. If $\mathcal{U}$ is convergent, by Corollary $12.1 \mathcal{I}(\mathcal{U})$ is of codimension 1 . Now, suppose that $\mathcal{U}$ is not convergent. There exists $\rho>0$ such that $\operatorname{diam}(X) \geq \rho$ for every $X \in \mathcal{U}$. Let $r \in] 0, \rho[$. Consider the covering of $E$ by the family of disks $\left(d_{E}\left(a_{i}, r^{-}\right)\right)_{i \in J}$ that are pairwise disjoint. Since $\operatorname{card}(E, r) \leq \operatorname{card}(U, r)$, there exists a covering of $U$ by a family of disks $\left(d\left(b_{j}, r^{-}\right)_{i \in I}\right)$ pairwise disjoint, with an injection $\theta$ from $I$ to $J$. So, we have $\left|b_{\theta(i)}-b_{\theta(j)}\right| \leq r$ for every $i \neq j$. 
Consider now the function $f$ from $E$ to $\mathbb{K}$ defined by $f(x)=b_{\theta(i)}$ for every $x \in d_{E}\left(a_{i}, r^{-}\right)$. Let $X \in \mathcal{U}$. Since $\operatorname{diam}(X) \leq r$, there exists $a_{i}, a_{j} \in X$ with $a_{i} \neq a_{j}$ and we have $f\left(a_{i}\right)=b_{i}, f\left(a_{j}\right)=b_{j}$, hence $\left|f\left(a_{i}\right)-f\left(a_{j}\right)\right| \geq r$ which shows that $f$ does not converge on $\mathcal{U}$ and therefore, by Corollary 26.1, $\mathcal{I}(\mathcal{U})$ is not of finite codimension.

Corollary 30.1: $\quad$ Suppose that $E$ is separable and that $\mathbb{K}$ is not locally compact. Let $\mathcal{U}$ be a non-convergent ultrafilter on $E$. Then $\mathcal{I}(\mathcal{U})$ is of infinite codimension.

Remark 12: Particularly Theorem 30 applies when $\mathbb{K}$ is algebraically closed and $E$ is separable.

Let us recall some results on the Shilov boundary of an ultrametric normed algebra [6].

Given a norm of $\mathbb{K}$-algebra, we call Shilov boundary of $T$ a closed subset $S$ of $\operatorname{Mult}(T,\|\|$.$) that is minimum with respect to inclusion, such that, for every$ $x \in T$, there exists $\phi \in S$ such that $\phi(x)=\|x\|_{s i}$.

Proposition G [3], [5] : Every normed $\mathbb{K}$-algebra admits a Shilov boundary.

Theorem 31: The Shilov boundary $S$ of $\mathcal{D}(E)$ is equal to $\operatorname{Mult}(\mathcal{D}(E), \|$. $\|)$.

Proof. Henceforth we take $\epsilon \in] 0, \frac{1}{2}$ [. Suppose that the Shilov boundary $S$ of $\mathcal{D}(E)$ is not equal to $\operatorname{Mult}(\mathcal{D}(E),\|\|$.$) and let \psi \in \operatorname{Mult}(\mathcal{D}(E), \|$. $\|) \backslash S$. Since $S$ is a closed subset of $\operatorname{Mult}(\mathcal{D}(E),\|\cdot\|)$, the set $\operatorname{Mult}(\mathcal{D}(E),\|\cdot\|) \backslash S$ is an open subset of $\operatorname{Mult}(\mathcal{D}(E),\|\cdot\|)$ and hence, there exist $f_{1}, \ldots, f_{q}$ such that $W\left(\psi, f_{1}, \ldots, f_{q}, \epsilon\right) \subset$ $(\operatorname{Mult}(\mathcal{D}(E),\|\cdot\|) \backslash S)$. Let $L=\left\{x \in E|| \psi\left(f_{j}\right)-\mid f\left({ }_{j}(x)||_{\infty} \leq \frac{\epsilon}{2}\right\}\right.$. By Theorem 6, $L$ is uniformly open in $E$. Consequently, by Theorem 5 the characteristic function $u$ of $L$ belongs to $\mathcal{D}(E)$ and obviously satisfies $\psi(u)=1$. On the other hand, we have $u(x)=0$ for every $x \notin L$.

Now, there exists $\theta \in S$ such that $\theta(u)=\|u\|=1$. Consider the neighborhood $W\left(\theta, f_{1}, \ldots, f_{q}, u, \frac{\epsilon}{2}\right)$. Since $\operatorname{Mult}_{E}(\mathcal{D}(E), \|$. $\|)$ is dense in $\operatorname{Mult}(\mathcal{D}(E),\|\|$.$) , we$ can take $\varphi_{a} \in W\left(\theta, f_{1}, \ldots, f_{q}, u, \frac{\epsilon}{2}\right)$ we have ||$u(a)|-\theta(u)|_{\infty} \leq \frac{\epsilon}{2}$, hence $|u(a)| \geq$ $1-\frac{\epsilon}{2}>0$. But since $\theta \in S$, we have $W\left(\theta, f_{1}, \ldots, f_{q}, \frac{\epsilon}{2}\right) \cap W\left(\psi, f_{1}, \ldots, f_{q}, \frac{\epsilon}{2}\right)=\emptyset$ and so much the more: $W\left(\theta, f_{1}, \ldots, f_{q}, u, \frac{\epsilon}{2}\right) \cap W\left(\psi, f_{1}, \ldots, f_{q}, u, \frac{\epsilon}{2}\right)=\emptyset$. Let $H=\{a \in$ $\left.E \mid \varphi_{a} \in W\left(\theta, f_{1}, \ldots, f_{q}, u, \frac{\epsilon}{2}\right)\right\}$. Then $H \cap L=\emptyset$. But by definition of $u$, we have $u(a)=0$ for every $a \in H$, a contradiction.

\section{Morphisms between algebras of strictly differentiable functions}

Notations: Henceforth, we denote by $E^{\prime}$ another open subset of $\mathbb{I K}$ and by $\mathcal{D}\left(E^{\prime}\right)$ the $\mathbb{K}$-algebra of strictly differentiable functions from $E^{\prime}$ to $\mathbb{K}$ whose norm is denoted by $\|.\|^{\prime}$. We denote by $\gamma$ an injective strictly differentiable function from $E^{\prime}$ to $E$ and for every $f \in \mathcal{D}\left(E^{\prime}\right)$, we set $\widetilde{\gamma}(f)=f \circ \gamma$.

Theorem 32: Let $f \in \mathcal{D}(E)$. Then $f \circ \gamma$ belongs to $\mathcal{D}\left(E^{\prime}\right)$ and $\widetilde{\gamma}$ is a morphism from $\mathcal{D}(E)$ to $\mathcal{D}\left(E^{\prime}\right)$ such that $\|\widetilde{\gamma}(f)-\widetilde{\gamma}(g)\|^{\prime} \leq\|f-g\|$ for every $f, g \in \mathcal{D}(E)$. 
Moreover, if $\gamma$ is a bijection from $E^{\prime}$ onto $E$, then $\widetilde{\gamma}$ is injective and particularly and if $\gamma^{-1}$ is also strictly differentiable, then $\widetilde{\gamma}$ is an isometric isomorphism.

Proof. Let $f \in \mathcal{D}(E)$. Let us first show that $\widetilde{\gamma}(f)$ belongs to $\mathcal{D}(E)^{\prime}$. Set $h=f \circ \widetilde{\gamma}$. Given $u, v \in E^{\prime}, u \neq v$, since $\gamma$ is injective, we can write

$$
\frac{h(u)-h(v)}{u-v}=\left(\frac{f(\gamma(u))-f(\gamma(v))}{\gamma(u)-\gamma(v)}\right)\left(\frac{\gamma(u)-\gamma(v)}{u-v}\right)
$$

By definition, the mapping $\phi$ from $(E \times E) \backslash D$ to $\mathbb{K}$ given by $\phi(x, y)=\frac{f(x)-f(y)}{x-y}$ is bounded and expands to a continuous function from $E \times E$ to $\mathbb{K}$. Similarly, denoting by $D^{\prime}$ the set $\left\{(u, u) \mid u \in E^{\prime}\right\}, \gamma$ is bounded in $\left(E^{\prime} \times E^{\prime}\right) \backslash D^{\prime}$ and expands to a continuous function from $E^{\prime} \times E^{\prime}$ to $\mathbb{K}$. Consequently, $h$ is bounded in $\left(E^{\prime} \backslash E^{\prime}\right) \backslash D^{\prime}$ and expands to a continuous function from $E^{\prime} \times E^{\prime}$ to $\mathbb{K}$. Therefore $h$ belongs to $\mathcal{D}(E)^{\prime}$. It is then obvious that $\|\widetilde{\gamma}(f)-\widetilde{\gamma}(g)\|^{\prime} \leq\|f-g\|$ for every $f, g \in \mathcal{D}(E)$. Suppose now that $\gamma$ is surjective. Let $f, g \in \mathcal{D}(E)$ be such that $\widetilde{\gamma}(f)=\widetilde{\gamma}(g)$. Then for all $u \in E^{\prime}$, we have $f(\gamma(u))=g(\gamma(u))$. But since $\gamma$ is surjective, for each $u \in E^{\prime}$, there exists $x \in E$ such that $x=\gamma(u)$, hence $f(u)=g(u)$ and hence $f=g$, which shows that $\widetilde{\gamma}$ is injective. Particularly, if $\gamma^{-1}$ is also strictly differentiable, then $\widetilde{\gamma}$ is an isomorphism from $\mathcal{D}(E)$ onto $\mathcal{D}\left(E^{\prime}\right)$ such that $\|\widetilde{\gamma}(f)-\widetilde{\gamma}(g)\|^{\prime}=\|f-g\|$ for every $f, g \in \mathcal{D}(E)$.

Notation: Let $\mathcal{H}$ be a class of contiguity on $U l\left(E^{\prime}\right)$. By Lemma M, we know that two contiguous ultrafilters on $E^{\prime}$ have images by $\gamma$ that are also contiguous. We denote by $\bar{\gamma}(\mathcal{H})$ the class of contiguity of the $\bar{f}(\mathcal{U}), \quad \mathcal{U} \in \mathcal{H}$.

Theorem 33: Let $\psi$ be a continuous morphism from $\mathcal{D}(E)$ to $\mathcal{D}\left(E^{\prime}\right)$ and let $\mathcal{M}^{\prime}$ be a maximal ideal of $\mathcal{D}\left(E^{\prime}\right)$. Then, $\psi^{-1}\left(\mathcal{M}^{\prime}\right)$ is a maximal ideal of $\mathcal{D}(E)$.

Proof. We know that $\psi^{-1}\left(\mathcal{M}^{\prime}\right)$ is a prime ideal of $\mathcal{D}(E)$. Since $\psi$ is continuous and since $\mathcal{M}^{\prime}$ is closed, $\psi^{-1}\left(\mathcal{M}^{\prime}\right)$ is closed, hence it is a closed prime ideal of $\mathcal{D}(E)$. Then by Corollary $14.2, \psi^{-1}\left(\mathcal{M}^{\prime}\right)$ is a maximal ideal of $\mathcal{D}(E)$.

Corollary 33.1: For every class of contiguity $\mathcal{H}^{\prime}$ on $E^{\prime}$, the maximal ideal $\mathcal{M}^{\prime}=$ $\mathcal{I}\left(\mathcal{H}^{\prime}\right)$ satisfies $(\widetilde{\gamma})^{-1}\left(\mathcal{M}^{\prime}\right)=\mathcal{I}\left(\bar{\gamma}\left(\mathcal{H}^{\prime}\right)\right)$.

Theorem 34: $\quad \gamma$ is supposed to be a strictly differentiable bijection from $E^{\prime}$ onto $E$. Then $\bar{\gamma}$ defines a surjective mapping from the set of the classes of contiguity on $E^{\prime}$ onto the set classes of contiguity on $E$ and $(\widetilde{\gamma})^{-1}$ defines a surjection from $\operatorname{Max}\left(\mathcal{D}\left(E^{\prime}\right)\right)$ onto $\operatorname{Max}(\mathcal{D}(E))$ as $(\widetilde{\gamma})^{-1}\left(\mathcal{I}\left(\mathcal{H}^{\prime}\right)\right)=\mathcal{I}\left(\bar{\gamma}\left(\mathcal{H}^{\prime}\right)\right)$.

Proof. Let $\mathcal{H}^{\prime}$ be a class of contiguity on $E^{\prime}$ and let $\mathcal{M}^{\prime}=\mathcal{I}\left(\mathcal{H}^{\prime}\right)$. By Lemma $\mathrm{M} \bar{\gamma}\left(\mathcal{H}^{\prime}\right)$ is a class of contiguity on $E$, hence $\mathcal{I}\left(\bar{\gamma}\left(\mathcal{H}^{\prime}\right)\right)$ is a maximal ideal of $\mathcal{D}(E)$. And by Corollary $14.2, \widetilde{\gamma}^{-1}\left(\mathcal{M}^{\prime}\right)$ is a maximal ideal of $\mathcal{D}(E)$, hence $\widetilde{\gamma}^{-1}\left(\mathcal{M}^{\prime}\right)$ is of the form $\mathcal{I}(\mathcal{H})$ where $\mathcal{H}$ is a class of contiguity of $E$. Consequently, $\mathcal{I}\left(\bar{\gamma}\left(H^{\prime}\right)\right)=\mathcal{I}(H)$. But since the mapping $\mathcal{I}$ defines a bijection from the set of classes of contiguity on $E$ onto $\operatorname{Max}(\mathcal{D}(E))$, we have $\bar{\gamma}\left(\mathcal{H}^{\prime}\right)=\mathcal{H}$ and hence $(\widetilde{\gamma})^{-1}\left(\mathcal{M}^{\prime}\right)=\mathcal{I}\left(\bar{\gamma}\left(\mathcal{H}^{\prime}\right)\right)$. 
Notation: Let $(T,\|\cdot\|)$ be a $\mathbb{K}$-normed algebra and let $\varphi \in M u l t(T,\|\cdot\|)$. Let $f_{1}, \ldots, f_{q} \in T$ and let $\left.\epsilon \in\right] 0,+\infty\left[\right.$. We will denote by $\mathcal{N}\left(\varphi, f_{1}, \ldots, f_{q}, \epsilon\right)$ the set $\left\{\phi \in \operatorname{Mult}(T,\|\cdot\|)|| \varphi\left(f_{k}\right)-\left.\phi\left(f_{k}\right)\right|_{\infty} \leq \epsilon\right\}$.

Recall that given a class of contiguity $\mathcal{H}$ on $E$ we denote by $\mathcal{I}(\mathcal{H})$ the maximal ideal $\mathcal{I}(\mathcal{U}), \mathcal{U} \in \mathcal{H}$. Similarly, given a class of contiguity $\mathcal{H}^{\prime}$ on $E^{\prime}$ we will denote by $\mathcal{I}^{\prime}\left(\mathcal{H}^{\prime}\right)$ the maximal ideal $\mathcal{I}\left(\mathcal{U}^{\prime}\right), \mathcal{U}^{\prime} \in \mathcal{H}^{\prime}$.

Theorem 35: Let $\gamma_{*}$ be the mapping from $\operatorname{Mult}\left(\mathcal{D}\left(E^{\prime}\right),\|\cdot\|^{\prime}\right)$ to $\operatorname{Mult}(\mathcal{D}(E), \| \cdot$ $\|)$ defined by $\left(\gamma_{*}\left(\varphi^{\prime}\right)\right)(f)=\varphi^{\prime}(f \circ \gamma),(f \in \mathcal{D}(E))$. Then $\gamma_{*}$ is continuous and surjective.

Proof. By Corollary 19.1 the mapping that associates to each $\varphi \in \operatorname{Mult}(\mathcal{D}(E), \| \cdot$ $\|)$ the ideal $\operatorname{Ker}(\varphi)$ is a bijection from $\operatorname{Mult}(\mathcal{D}(E),\|\cdot\|)$ onto $\operatorname{Max}(\mathcal{D}(E))$. Next, given $\varphi^{\prime} \in \operatorname{Mult}\left(\mathcal{D}\left(E^{\prime}\right),\|\cdot\|^{\prime}\right)$, by Corollary $19.2 \varphi^{\prime}$ is defined by a class of contiguity $\mathcal{H}^{\prime}$ on $E^{\prime}$ such that

$$
\varphi^{\prime}(f \circ \gamma)=\lim _{\mathcal{H}^{\prime}} f(\gamma(u))=\lim _{\bar{\gamma}\left(\mathcal{H}^{\prime}\right)}(f(x)) .
$$

Consequently, $\operatorname{Ker}\left(\varphi^{\prime}\right)=\mathcal{I}^{\prime}\left(\mathcal{H}^{\prime}\right)$ and $\operatorname{Ker}\left(\gamma_{*}\left(\varphi^{\prime}\right)\right)=\mathcal{I}(\mathcal{H})$.

Let us now show the continuity of $\gamma_{*}$. Consider again $\varphi^{\prime} \in \operatorname{Mult}\left(\mathcal{D}\left(E^{\prime}\right),\|\cdot\|^{\prime}\right)$ and a neighborhood $W=\mathcal{N}\left(\gamma_{*}\left(\varphi^{\prime}\right), f_{1}, \ldots, f_{q}, \epsilon\right)$ of $\gamma_{*}\left(\varphi^{\prime}\right)$ in $\operatorname{Mult}(\mathcal{D}(E),,\|\cdot\|)$ with $f_{j} \in \mathcal{D}(E), j=1, \ldots, q$. Set $g_{k}=f_{k} \circ \gamma, k=1, \ldots, q$ and let $V=\mathcal{N}\left(\varphi^{\prime}, f_{1}, \ldots, f_{q}, \epsilon\right)$ be a neighborhood of $\varphi^{\prime}$ in $\operatorname{Mult}\left(\mathcal{D}\left(E^{\prime}\right),\|\cdot\|^{\prime}\right)$. Then

$$
W=\left\{\psi \in \operatorname{Mult}(\mathcal{D}(E),\|\cdot\|)|| \psi\left(f_{k}\right)-\left.\left(\gamma_{*}\left(\varphi^{\prime}\right)\right)\left(f_{k}\right)\right|_{\infty} \leq \epsilon, k=1, \ldots, q\right\}
$$
and

$$
\begin{aligned}
& =\left\{\phi \in \operatorname{Mult}\left(\mathcal{D}\left(E^{\prime}\right),\|\cdot\|^{\prime}\right)|| \phi\left(g_{k}\right)-\left.\left(\varphi^{\prime}\right)\left(g_{k}\right)\right|_{\infty} \leq \epsilon, k=1, \ldots, q\right\} \\
& =\left\{\phi \in \operatorname{Mult}\left(\mathcal{D}\left(E^{\prime}\right),\|\cdot\|^{\prime}\right)|| \phi\left(f_{k} \circ \gamma\right)-\left.\left(\varphi^{\prime}\right)\left(f_{k} \circ \gamma\right)\right|_{\infty} \leq \epsilon, k=1, \ldots, q\right\} \\
& =\left\{\phi \in \operatorname{Mult}\left(\mathcal{D}\left(E^{\prime}\right),\|\cdot\|^{\prime}\right)||\left(\gamma_{*}(\phi)\right)\left(f_{k}\right)-\left.\left(\gamma_{*}\left(\varphi^{\prime}\right)\right)\left(f_{k}\right)\right|_{\infty} \leq \epsilon, k=1, \ldots, q\right\} \\
& =\left\{\phi \in \operatorname{Mult}\left(\mathcal{D}\left(E^{\prime}\right),\|\cdot\|^{\prime}\right) \mid\left(\gamma_{*}(\phi)\right) \in W\right\}, \\
& \text { hence } \gamma_{*}(V) \subset W . \text { That shows the continuity of } \gamma_{*} .
\end{aligned}
$$

Remark 13: We can derive the following interpretation: let $\varphi^{\prime} \in \operatorname{Mult}\left(\mathcal{D}\left(E^{\prime}\right), \|\right.$. $\|^{\prime}$ ) and let $\mathcal{M}^{\prime}=\operatorname{Ker}\left(\varphi^{\prime}\right)$. Then $(\widetilde{\gamma})^{-1}\left(\mathcal{M}^{\prime}\right)$ is a maximal ideal $\mathcal{M}$ of $\mathcal{D}(E)$ which is the kernel of a unique $\varphi \in \operatorname{Mult}(\mathcal{D}(E),\|\cdot\|)$. Consequently, $\varphi$ is defined as $\varphi(f)=\varphi^{\prime}(f \circ \gamma)$ described by Theorem 34 .

Acknowledgements: We are very grateful to the referee for pointing out to us many misprints.

\section{References}

[1] Escassut, A. Spectre maximal d'une algèbre de Krasner. Colloquium Mathematicum (Wroclaw) XXXVIII2, pp 339-357 (1978).

[2] Escassut, A. The ultrametric spectral theory, Periodica Mathematica Hungarica, Vol.11, (1), p7-60, (1980)

[3] Escassut, A. Analytic Elements in p-adic Analysis, World Scientific Publishing Co (1995).

[4] Escassut, A. Ultrametric Banach algebras. World Scientific Publishing Co (2003).

[5] Escassut, A. and Maïnetti, N. Spectral semi-norm of a p-adic Banach algebra, Bulletin of the Belgian Mathematical Society, Simon Stevin, vol 8, p.79-61, (1998).

[6] Escassut, A. and Maïnetti, N. Shilov boundary for ultrametric algebras, p-adic Numbers in Number Theory, Analytic Geometry and Functional Analysis, Belgian Mathematical Society, p.81-89, (2002) 
[7] Escassut, A. and Maïnetti, N. On Ideals of the Algebra of p-adic Bounded Analytic Functions on a Disk Bull. Belg. Math. Soc. Simon Stevin 14, p. 871-876 (2007)

[8] Escassut, A. and Maïnetti, N. About the Ultrametric Corona Problem Bulletin des Sciences Mathématiques 132, p. 382-394 (2008).

[9] Escassut, A. Ultrametric corona problem and sphericaly complete fields. Proceedings of the Edinburgh Mathematical Society (Series 2), Volume 53, Issue 02, June 2010, pp 353-371 (2010).

[10] Escassut, A. and Maïnetti, N. Multiplicative spectrum of ultrametric Banach algebras of continuous functions Topology and its applications 157, p. 2505-25015 (2010).

[11] Escassut, A. and Maïnetti, N. Morphisms between ultrametric Banach algebras and maximal ideals of finite codimension Contemporary Mathematics 596, p. 63-71 (2013)

[12] Escassut, A. Value Distribution in p-adic Analysis. WSCP Singapore, (2015)

[13] Garandel, G. Les semi-normes multiplicatives sur les algèbres d'éléments analytiques au sens de Krasner, Indag. Math., 37, n4, p.327-341, (1975).

[14] Guennebaud, B. Sur une notion de spectre pour les algèbres normées ultramétriques, thèse d'Etat, Université de Poitiers, (1973).

[15] Haddad, L. Sur quelques points de topologie générale. Théorie des nasses et des tramails. Annales de la Faculté des Sciences de Clermont N 44, fasc.7, p.3-80 (1972)

[16] Schikhof, W. Ultrametric calculus. An Introduction to p-adic analysis, Cambridge University Press, Cambridge, (1984).

[17] Van der Put, M. The Non-Archimedean Corona Problem. Table Ronde Anal. non Archimedienne, Bull. Soc. Math. Mémoire 39-40, p. 287-317 (1974).

Université Clermont Auvergne,, Laboratoire de Mathématiques Blaise Pascal, UMR-CNRS, BP 10448, F-63000 CLERMONT-FERRAND, FRANCE.

E-mail address: alain.escassut@math.univ-bpclermont.fr

département Gea iUt d’ Aurillac, Université Clermont Auvergne, 100 Rue de L'ÉGALITÉ, 15000 AURILLAC

E-mail address: Nicolas.Mainetti@iut.u-clermont1.fr 